\title{
Smart Polymeric Delivery System for Antitumor and Antimicrobial Photodynamic Therapy
}

\author{
Zhijia Wang, Fu-Jian Xu and Bingran Yu* \\ Laboratory of Biomedical Materials and Key Lab of Biomedical Materials of Natural Macromolecules Beijing University of Chemical \\ Technology, Ministry of Education, Beijing University of Chemical Technology, Beijing, China
}

OPEN ACCESS

Edited by:

Yakai Feng,

Tianjin University, China

Reviewed by:

Wull Yang,

Fudan University, China

Dong Wang

Shenzhen University, China

*Correspondence:

Bingran Yu

yubr@mail.buct.edu.cn

Specialty section:

This article was submitted to Biomaterials,

a section of the journal

Frontiers in Bioengineering and

Biotechnology

Received: 26 September 2021

Accepted: 14 October 2021

Published: 04 November 2021

Citation:

Wang Z, Xu F-J and Yu B (2021) Smart

Polymeric Delivery System for

Antitumor and Antimicrobial

Photodynamic Therapy.

Front. Bioeng. Biotechnol. 9:783354.

doi: 10.3389/fbioe.2021.783354
Photodynamic therapy (PDT) has attracted tremendous attention in the antitumor and antimicrobial areas. To enhance the water solubility of photosensitizers and facilitate their accumulation in the tumor/infection site, polymeric materials are frequently explored as delivery systems, which are expected to show target and controllable activation of photosensitizers. This review introduces the smart polymeric delivery systems for the PDT of tumor and bacterial infections. In particular, strategies that are tumor/bacteria targeted or activatable by the tumor/bacteria microenvironment such as enzyme/pH/ reactive oxygen species (ROS) are summarized. The similarities and differences of polymeric delivery systems in antitumor and antimicrobial PDT are compared. Finally, the potential challenges and perspectives of those polymeric delivery systems are discussed.

Keywords: polymer, target, activatable, photodynamic therapy, antibacterial

\section{INTRODUCTION}

Photodynamic therapy (PDT) has attracted intensive attention for the treatment of tumor and bacteria during recent years(Celli et al., 2010; Bugaj, 2011; Kamkaew et al., 2013; Lincoln et al., 2013; Tian et al., 2013). For instance, for traditional cancer therapy strategies such as chemotherapy and radiotherapy, patients often suffer from severe side effects, limited tumor suppression, and unavoidable regrowth of metastatic tumor. In comparison, PDT has high spatiotemporal selectivity in triggering tumor cell death and can generate immune response (Wang et al., 2020), showing advantages over the traditional cancer therapies. On the other hand, concerning antibacterial therapy, antibiotic resistance problems become increasingly prominent (Maisch et al., 2004). PDT is expected to be a promising alternative to antibiotic therapy for combating bacterial infections (Klausen et al., 2020). PDT therapeutics such as Porfimer sodium, ALA, and Verteporfin have been approved worldwide to be used in clinics (Agostinis et al., 2011). PDT has been approved to treat some diseases in clinical trials, such as premalignant tumors, cutaneous malignant tumors, tumors of the head, neck, and oral cavity, lung, gastrointestinal, and other tumors (Brown et al., 2004), viral lesions, acne, gastric infection by Helicobacter pylori, and brain abcesses (Hamblin and Hasan, 2004).

The mechanism of PDT is described in Figure 1. With light irradiation, the photosensitizer is promoted to its singlet excited state, followed by intersystem crossing to its triplet state. As the transition from triplet excited state $\left(T_{1}\right)$ to ground state is spin forbidden, this $T_{1}$ state is relatively long-lived and can react with molecular oxygen. Concerning the reaction type between the $\mathrm{T}_{1}$ state and molecular oxygen, there are two possibilities. Electron transfer between the $T_{1}$ state and molecular oxygen will initially produce a superoxide anion $\left(\mathrm{O}_{2}{ }^{--}\right)$, and subsequently produce other 


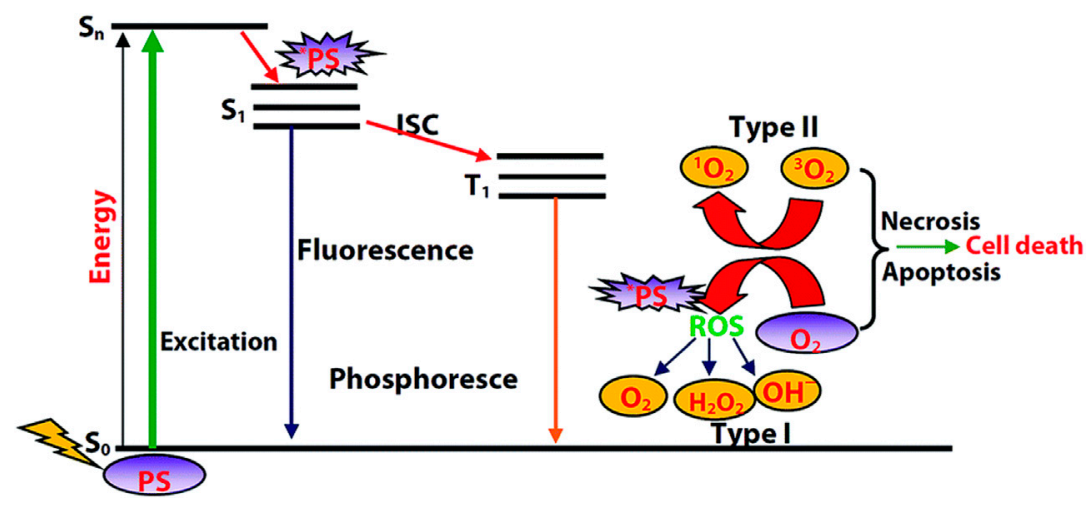

FIGURE 1 | Diagram illustrating the process of PDT. Reprinted with permission from Majumdar et al. (2014).

ROS such as hydroxyl radical or hydrogen peroxide that is toxic to cells or bacteria, which is the so-called Type I PDT. Energy transfer between the $\mathrm{T}_{1}$ state and molecular oxygen will produce toxic singlet oxygen $\left({ }^{1} \mathrm{O}_{2}\right)$ to kill tumor cells or bacteria, which is Type II PDT.

It is obvious that triplet photosensitizer, light, and oxygen are three key factors of PDT. Among these, triplet photosensitizer plays a leading role (Majumdar et al., 2014). Although a variety of conventional photosensitizers such as porphyrin derivatives (e.g., chlorin e6, PpIX), phthalocyanine derivatives (e.g., $\mathrm{ZnPc}$ ), and some newly developed triplet photosensitizers such as Bodipy derivatives have PDT ability (Detty et al., 2004), their water solubility is unsatisfactory. The delivery of those photosensitizers to tumor or infected site is a critical issue.

Polymers consist of many structural units or monomers that are connected with a covalent bond; hence, they have a super high molecular weight. Some polymers play the role of triplet photosensitizers as they can produce ROS (Blacha-Grzechnik, 2021). Some reports found that polymers may have enhanced ROS production ability than monomers, which might be due to the increased number of vibrational energy levels and broader energy bands of polymers as compared with monomers (Wu et al., 2018c; Liu et al., 2021). More commonly, polymers functioned as carries of organic triplet photosensitizer, which increases its water solubility and its accumulation in the tumor or infected site (Regehly et al., 2007; Lee et al., 2009; Park S. Y. et al., 2011; Huang et al., 2016; Cui et al., 2019). In addition, small molecules tend to be cleaned up quickly from the body; hence, the polymers are expected to extend the circulation time. Polymers can also serve as a gene delivery vector, e.g., the delivery of a genetically encoded photosensitizer (such as killerred, miniSOG) for PDT cancer treatment (Sarkisyan et al., 2015; Tseng et al., 2015). Polymers have versatile functions and can combine with other therapy strategies, achieving multimodal therapy ( $\mathrm{Li}$ and $\mathrm{Pu}, 2020)$. For instance, polymers can act as prodrugs for PDT and chemotherapy (Xu et al., 2016; Wang et al., 2019; Lu L. et al., 2020), the combination of polymer and cytotoxic enzymes shows outstanding tumor treatment efficacy (Li et al., 2019c); it can also function as a blockader to intervene in the protein biosynthesis to depress the tumor ( $\mathrm{Li}$ et al., 2019b); the polymers become stimulants in the combination of PDT and immunotherapy (Li et al., 2019a)

Smart polymers that can target tumor/infected tissues or be activatable by the tumor/bacterial microenvironment are highly desired. Although the PDT has relatively high spatial selectivity, there is still great concern about the skin phototoxicity. For instance, patients treated with porphyrin derivatives have to stay in the dark for at least $72 \mathrm{~h}$, which is a great psychological challenge to patients. An activatable delivery system is beneficial to reduce the skin phototoxicity. Small organic molecules tend to be cleared out from the body, and an activatable polymeric delivery system can protect the small photosensitizer and achieve on site release of photosensitizer. Moreover, the nonspecifically distribution of the triplet photosensitizer in the whole body requires a higher dose of photosensitizer, which is more likely to lead to organ damage; a specific accumulation of the photosensitizer to the lesion site is beneficial to reduce administration dosage, elevate the therapeutic efficacy, and play down the side effects. Hence, developing smart polymeric delivery systems that can target the tumor/bacterial site or activate by the tumor/bacterial microenvironment is urgent. In recent years, elegant works have been conducted in these areas (Hoffman, 2008; Li and Huh, 2014; Kamaly et al., 2016; Chen et al., 2017; Wu L. et al., 2018; Wu et al., 2018b; Li and Yan, 2018; Li et al., 2020; Zheng et al., 2020; Han et al., 2021). This review will give a brief summary on the smart polymeric delivery system for antitumor and antimicrobial photodynamic therapy. Note that due to the limitation of the space, we only selected part of typical articles as examples to introduce in detail. The authors apologize for omitting some important and elegant works in advance.

\section{SMART POLYMERIC DELIVERY SYSTEM FOR ANTITUMOR PHOTODYNAMIC THERAPY}

Cancer has been a leading cause of death all over the world (Ferlay et al., 2010). Data from the World Health Organization (WHO) 
shows that nearly 10 million deaths from cancer occurred in 2020. Traditional cancer therapy strategies such as chemotherapy and radiotherapy have severe side effects (Mitsunaga et al., 2011; Wang and Guo, 2013; Lucky et al., 2015) Moreover, there are great concerns on the drug resistance and radioactivity for chemotherapy and radiotherapy, respectively (Mitsunaga et al., 2011; Wang and Guo, 2013; Lucky et al., 2015).

PDT has attracted tremendous attention since its birth in the early 1900s, when Tappeiner conducted the first therapy to himself by topical eosin and sunlight. Later, Figger et al. and Lipson et al. discovered the photosensitizer hematoporphyrin and forwarded the PDT of the tumor to clinical applications, respectively (Figge et al., 1948). Compared with conventional approaches of cancer therapy such as surgical excision, chemotherapy, and radiotherapy, PDT shows great advantages such as higher spatial/temporal resolution and the anti-drug resistance.

As the photosensitizers may distributed over the whole body, a smart polymeric delivery system is beneficial for precise therapy. The delivery system is expected to protect the photosensitizers from systemic clearance, and carry the photosensitizer to the tumor site. In some cases, the delivery system releases the photosensitizers, either with a slow sustained release or a burst of release. Hence, the sustained release and biodegradable polymeric delivery systems are introduced. Smart polymeric delivery systems that can target the tumor and be activatable by the tumor microenvironment are highlighted.

\section{Sustained Release and Biodegradable Polymeric Delivery System}

One of the most crucial functions of a polymeric delivery system is to enhance the water solubility of hydrophobic photosensitizers. For instance, polyethylene glycol (PEG) is one of the most frequently used polymers to increase the water solubility. PEG provides stealth to photosensitizers, making them invisible to microorganisms and cells. Hence the protection by PEG resisted protein adsorption and prolonged the circulatory half-life of photosensitizer (Keerthiga et al., 2020), which is beneficial to achieve long-term antitumor effects.

Polymers with high molecular weight may raise great concern about their toxicity. Hence, it is highly desired that the polymer can be degraded into small nontoxic fragments such as water and carbon dioxide, which is easy to be metabolized from the body (Kamaly et al., 2016). Concerning this aspect, biodegradable polymers such as polylactide (PLA), poly (glycolic acid) (PGA), poly (lactic-co-glycolic acid) (PLGA), and poly$\beta$-benzyl-L-aspartate (PBLA) have been developed, which can be degraded slowly by the hydrolysis of the ester bond (Vert et al., 1992; Hoffman, 2008; Kim et al., 2009; Keerthiga et al., 2020) Hence the encapsulated photosensitizers can be sustained released.

\section{Tumor-Target Polymeric Delivery System Passive Targeting}

The passive targeting of those polymeric nanoparticles is referred to the enhanced permeation and retention (EPR) effect proposed by Hiroshi Maeda in Kumamoto, Japan, with the theory based on the high permeability of blood vessels at tumor sites, due to the leakage of the tumor vasculature as a consequence of growing fast (Hoffman, 2008). There are plenty of systems that enhance the accumulation efficacy to tumor via EPR effect (Regehly et al., 2007; Lee et al., 2009). However, this targeting strategy may release a considerable quantity of drugs before substantial uptake by tumor cells. Moreover, recently the EPR effect is becoming a controversial topic as researchers found that human bodies do not have obvious leakage of tumor vasculature and EPR effect. Hence, the general applicability of this EPR effect is challenged and still an open question.

\section{Active Targeting}

Active targeting includes the use of targeting moieties such as ligands, antibodies, and aptamers that can recognize specific receptors on tumor vasculature, tumor cells, and even tumor subcellular organelles for enhanced delivery of photosensitizers (Li and Yan, 2018; Zhang et al., 2020; Zheng et al., 2020)

The tumor vasculature targeting is an efficient way to combat cancer as a result of cutting off the supply of oxygen and nutrients. In addition, by interrupting the tumor vessel integrity, an increased accumulation of the materials to the tumor tissue is expected to improve the PDT efficacy. Vascular endothelial growth factor (VEGF) and its receptor are hallmarks of tumor cells, which can be utilized as main antiangiogenic targets (Tirand et al., 2006; Prasad et al., 2014). Vascular cell adhesion molecule-1 (VCAM-1) is involved in tumor cell adhesion and metastasis (Yin et al., 2017), and matrix metalloproteinases (MMPs) are responsible for the angiogenesis and metastasis (Danhier et al., 2010; Liu T. W. et al., 2016), RGD ligands are another frequently used target and the recognition is via $\alpha_{v} \beta_{3}$ integrin (Yuan et al., 2014). All of them are crucial tumor vasculature targets.

Target tumor cells are a straightforward targeting strategy that accelerate the phagocytosis/endocytosis of nanocarriers (Hanahan and Weinberg, 2011; Liu L. et al., 2016; Liu et al., 2017). The representative receptors are CD44 receptor (Jiang et al., 2017), folate (FA) receptor (Li et al., 2018d), transferrin receptor (Kaspler et al., 2016), epidermal growth factor (EGF) receptor (del Carmen et al., 2005), etc.

Subcellular targeting, such as mitochondria, lysosome, nucleus, and endoplasmic reticulum, is another type of targeting. Among these, mitochondria targeting is of great importance as mitochondria is the energy factory of tumor cells (Chakrabortty et al., 2017). The triphenylphosphonium (TPP) cation is a typical mitochondria targeting moiety. Due to the limited diffusion distance of singlet oxygen, the subcellular targeting of different organelles may lead to varying degrees of destruction of tumor cells.

With a large amount of targeting candidates mentioned above, herein we only choose one example for the demonstration of the importance of targeting function in PDT. Target function is especially crucial to cross the blood-brain-barrier (BBB) in the treatment of brain tumor. The treatment of brain disease is not a trivial task as the delivery of the drugs to the brain area is very difficult due to the BBB. An iRGD-conjugated prodrug micelle 
A

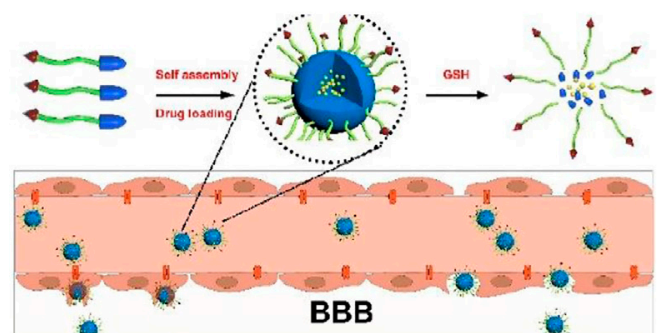

.
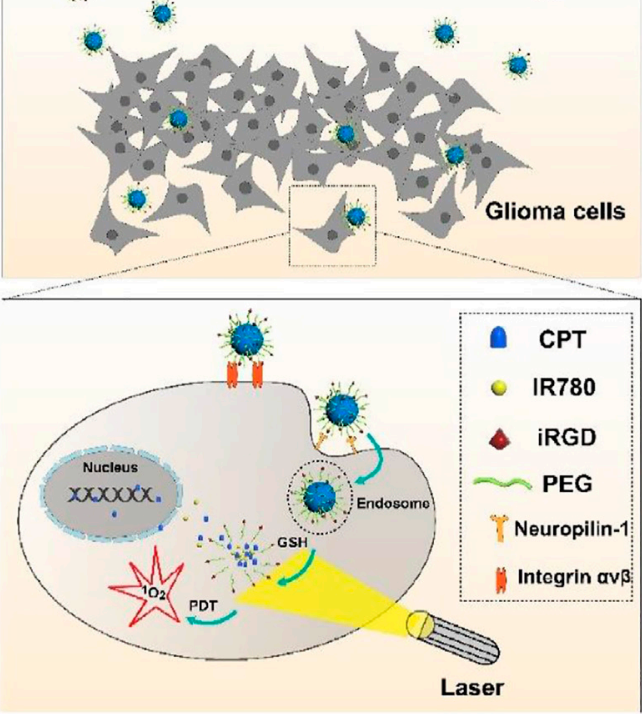

B

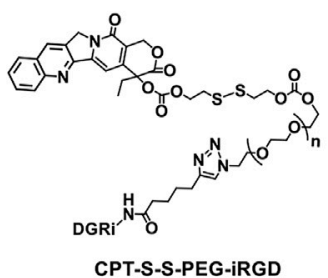

D
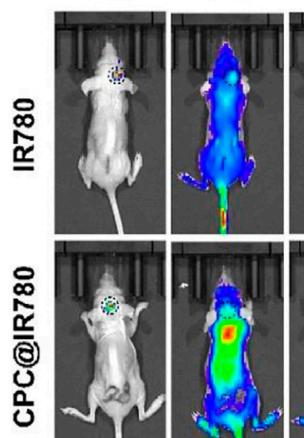

C

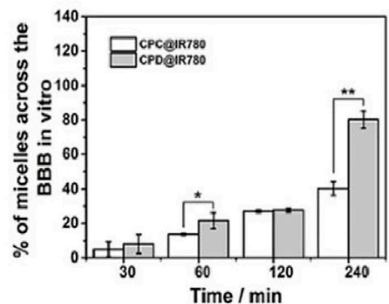

$6 \mathrm{~h} \quad 12 \mathrm{~h} \quad 24 \mathrm{~h}$
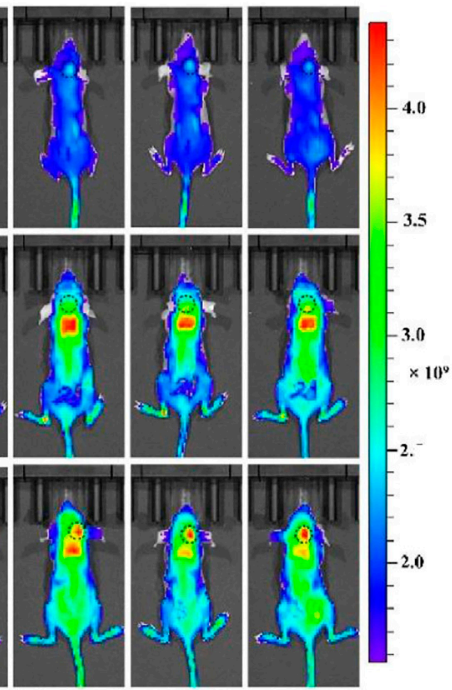

E Merge $\quad 0 \mu \mathrm{m}$ $45 \mu \mathrm{m}$ $90 \mu \mathrm{m}$

$135 \mu \mathrm{m}$

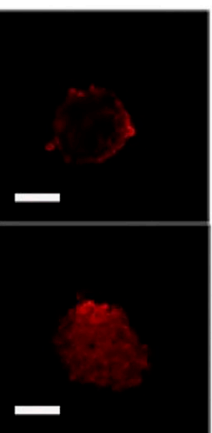

FIGURE 2 | (A) Preparation of self-assembled micelles, BBB penetrating, and glioma cell targeting by the iRGD peptide and GSH-induced disassembly for the combination of chemotherapy and photodynamic therapy; (B) molecular structure of CPT-S-S-PEG-iRGD; (C) the quantitative analysis of absorption spectra in the lower chamber after CPC@IR780 micelles and CPD@|R780 micelles were cultured in the upper chamber for different times; (D) whole body fluorescence imaging of U87 orthotopic glioma mice at different time intervals. Dashed line indicates the glioma site; (E) intratumoral penetration characterization using U87 tumor spheroids treated with micelles in $45 \mu \mathrm{m}$ interval between consecutive slides. Reprinted with permission from Lu L. et al. (2020).

with $\mathrm{BBB}$ penetrability was used to enhance the anti-glioma therapy (Lu L. et al., 2020) As shown in Figure 2, the structure of the polymer CPT-S-S-PEG-iRGD consists of a prodrug camptothecin (CPT) for chemotherapy, a disulfide linker responsive to glutathione (GSH), and the internalizing RGD peptide (iRGD) as targeting moiety. The ability to cross the $\mathrm{BBB}$ and target glioma cells is expected to be enhanced via av $\beta$ integrin and neuropilin-1 mediated ligand transportation. The polymer is self-assembled into micelle with a diameter of approximately $100 \mathrm{~nm}$. A photosensitizer IR780 is loaded into the micelle for PDT.

Hence, the micelle is functionalized as the CPT and photosensitizer IR780 carrier, which is responsive to the high concentration of GSH in glioma tumor and triggers the release of the therapeutics CPT and IR780. The iRGD peptide bonded at the surface of the micelles is responsible for the cross of the BBB and glioma tumor targeting. The penetration ability of the micelles is examined in vitro BBB model, which is established by co-culture 
of bEnd. 3 cells and U87 cells in the upper and lower chamber, respectively. The bEnd. 3 simulates the $\mathrm{BBB}$. By examination of the uptake efficiency of the micelles by U87 cells in the lower chamber, the penetration ability of the materials is found to be significantly improved (Figure 2C). The results show that $89.92 \pm$ $1 \%$ of iRGD modified CPD@IR780 micelles cross the simulated BBB into the lower chamber, while for the unmodified CPC@ IR780 micelles the value is only $41.78 \pm 0.22 \%$.

The authors further constructed U87 tumor spheroids in vitro to examine the penetration ability (Figure 2E). For the CPD@ IR780 micelles, the fluorescence is stronger and uniformly distributed in the glioma spheroids. While for the CPC@IR780 micelles, the fluorescence is mainly distributed in the periphery of the glioma spheroids. These results demonstrated the enhanced penetration ability to the glioma spheroids of CPD@IR780 micelles due to iRGD modification. The biodistribution of micelles in vivo was studied (Figure 2D). The iRGD modified CPD@IR780 micelles show enhanced accumulation in the brain, as compared to the unmodified CPC@IR780 micelles and IR780. Confocal images of brain sections of U87 orthotopic glioma mice were studied. The iRGD modified CPD@IR780 micelles show preferably distribution in glioma tumor cells instead of healthy cells. All those results confirmed the target ability of CPD@IR780 micelles to glioma cells. The combination of activatable chemotherapy and PDT with target function to enhance penetration to $\mathrm{BBB}$ successfully extended the survival time of mice bearing glioma tumor.

\section{Tumor-Activatable Polymeric Delivery System}

Abnormal expression of proteins usually has a close relationship with diseases. Enzymes are especially crucial biomarkers, and a variety of enzymes are overexpressed by tumor cells or tumor associated macrophages. For instance, hyaluronidase, matrixmetalloproteinases, and cathepsin B are overexpressed in tumor cells. The enzyme-activated polymeric delivery systems have been developed, which have high specificity as compared with other activation strategies such as $\mathrm{pH}$ and GSH activation (Bae and Na, 2010; Park W. et al., 2011; Li H. et al., 2017; Huo et al., 2019).

The increased glycolysis and proton-pump activities on plasma membranes of cancer cells produce a large amount of lactic acid, resulting in a slightly acidic extracellular microenvironment of tumor. The $\mathrm{pH}$ value of tumor sites is in the range of 6.0-7.0, which is lower than that of normal tissues $(\mathrm{pH}=7.4)$ (Pan et al., 2013). Taking advantage of the difference in $\mathrm{pH}$, an acid activatable system can be designed (Du et al., 2010; Du et al., 2011; Yuan et al., 2012; Tseng et al., 2015; Li F. et al., 2018; Dong et al., 2018; Lin et al., 2019; Lu Y. et al., 2020) mainly based on the protonation of the amine group or acid cleavage of acidic-labile linkers such as hydrazone linkers (Braslawsky et al., 1990), Schiff base linkers (-RC = N-) (Ke et al., 2014), cis-acotinyl linkers (Zhu et al., 2010), acetal linkers (Gu et al., 2013), etc. (Figure 3A).

Reactive oxygen species (ROS) and GSH are of great importance for maintaining the redox stability. Tumor cells have higher levels of ROS due to the oncogenic stimulation, mitochondrial malfunction, and increased metabolic activity $(\mathrm{Li}$ X. et al., 2017). For instance, the concentration of hydrogen peroxide in tumor cells is reported to be approximately $10 \mu \mathrm{M}$, which is higher than normal cells. Boronic acid esters are typical hydrogen peroxide-responsive moieties (Saravanakumar et al., 2017; Zeng et al., 2020). In addition, GSH is abundant in a tumor intracellular environment (2-10 mM) (Li and Yan, 2018). Disulfides and sulfonyl group are frequently utilized to construct GSH-responsive polymeric delivery systems (Kim et al., 2014; Li et al., 2014; Liu X. et al., 2016). The representative ROS and GSH responsive organic moieties are summarized in Figure 3A.

Due to the consumption of oxygen within about $100 \mu \mathrm{m}$ of the inadequate tumor vasculatures by quickly proliferating cancer cells, the oxygen concentration of tumors is only about $4 \%$, which is very distinct from normal tissues. Based on this, hypoxiaresponsive polymeric systems have been developed and three representative hypoxia-responsive moieties have been summarized in Figure 3A.

Based on the different microenvironments of tumor and normal tissues, a variety of activatable polymeric delivery systems are emerging (Park et al., 2016; Sun et al., 2017; Wei et al., 2018; Li et al., 2019c; Yan et al., 2020; Zeng et al., 2020; Yang et al., 2021). Despite the different structures and constituents of thousands of reports, they mainly achieve the activation via the following three strategies: (1) self-quenching and dequenching of photosensitizers due to aggregation and disintegration; (2) utilize another quencher to quench the triplet state of photosensitizer and dequenching upon cleavage of sensitive bonds; and (3) the change of size and surface charge to induce an enhanced internalization by tumor cells. These three strategies are summarized in Figure 3B.

Herein we introduce some typical polymeric delivery systems for PDT, as an illustration of the above-mentioned activations and strategies.

\section{Enzyme}

Choi et al. reported a protease biodegradable poly-L-lysine, grafted with PEG and photosensitizer Ce6 (L-SR15). As shown in Figure 4, the quenched fluorescence and singlet oxygen production ability is recovered due to the degradation of the poly-L-lysine by tumor-associated protease cathepsin B. A polyD-lysine backbone (D-SR16) that is uncleavable by proteases was synthesized for comparison. In vitro results are shown in Figures 4B,C, both the fluorescence/singlet oxygen generation ability of L-SR 15 and D-SR16 are significantly decreased as compared with the free Ce6. The fluorescence intensity/singlet oxygen generation ability of L-SR15 is recovered by the addition of cathepsin B, while there is no change for D-SR16. The fluorescence/singlet oxygen generation ability enhancement can be inhibited by the addition of cathepsin B inhibitor CA-074. In vivo results show that the tumor can be imaged by the L-SR15 (Figure 4D), due to the selectively activation by the cathepsin B in the tumor site. The tumor volume is significantly depressed for the L-SR15 group as compared with D-SR16 group or L-SR15 + inhibitor CA-074 group. These results demonstrated that the tumor-specific 

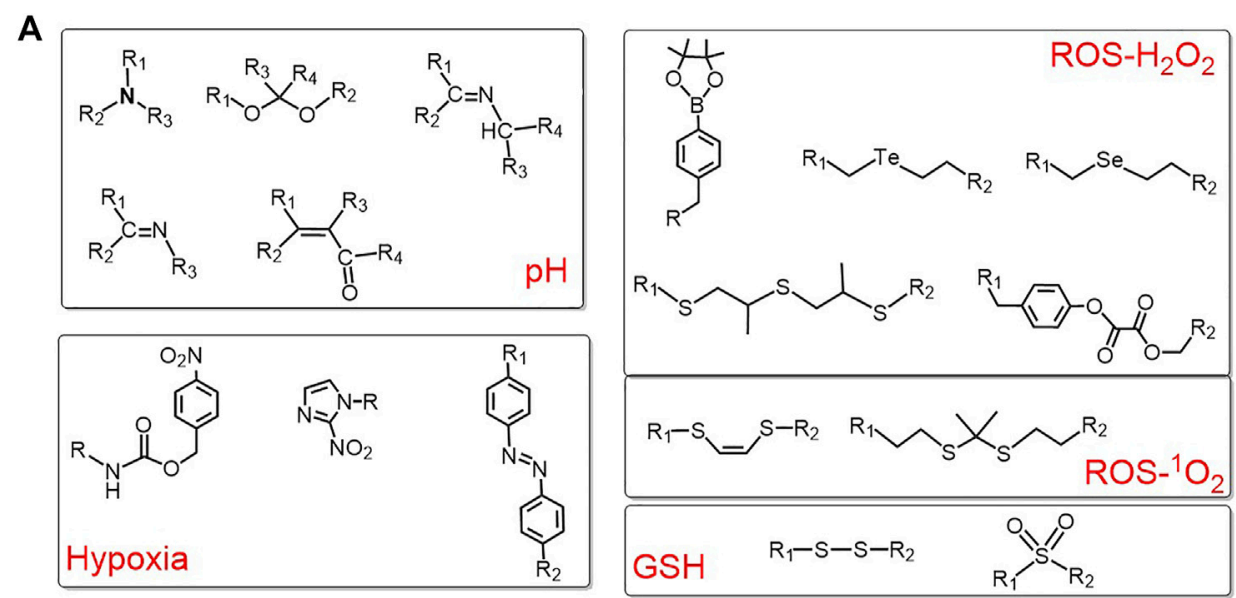

\section{B}

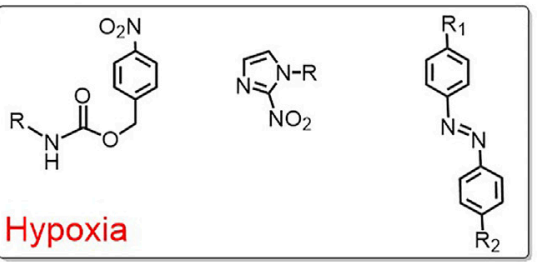

GSH

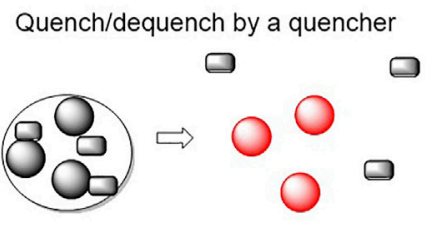

Size reduction and charge inversion

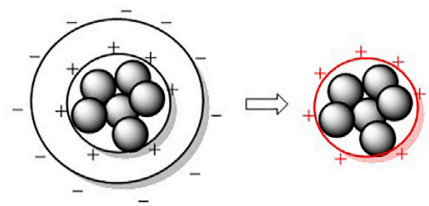

FIGURE 3 | (A) Representative pH/ROS/GSH/hypoxia-responsive linkers for activatable polymeric delivery system; (B) three commonly used activation strategies.

protease cleaved the poly-L-lysine backbone, restoring the selfquenched fluorescence/singlet oxygen production ability of Ce6 and achieving selective imaging and PDT of the tumor.

A similar strategy was reported by Kun $\mathrm{Na}$ et al., who is also one of the pioneer researchers in this area. For instance, photosensitizer Pheophorbide (Pba) was dangled onto hyaluronic acid (HA), which leads to self-quenching of the photosensitizer. The polymer can be easily internalized by HA receptor-mediated endocytosis and then degraded by intracellular microenvironment enzymes, releasing free $\mathrm{Pba}$ with recovered PDT efficacy (Li et al., 2010). However, those reports by Choi et al. and Kun $\mathrm{Na}$ et al. are based on the selfquenching of photosensitizers. Both the quenching and activation efficiency are highly dependent on the substitution degree of the photosensitizers to the polymer backbone. A higher degree of substitution will lead to higher quenching efficiency but maybe lower activation efficiency because it decreases the recognition site of the biomarker.

Zheng et al. reported a molecular beacon system based on intramolecular quenching (Zheng et al., 2007). As shown in Figure 5A, triplet photosensitizer (pyropheophorbide, Pyro) is connected with a quencher (BHQ3) by a peptide linker, which is activatable by tumor-associated protease MM7. Both the fluorescence and the singlet oxygen production ability of Pyro can be quenched by BHQ3, while it restored upon cleavage of the peptide linker by the MM7. This is confirmed with fluorescence emission and the direct detection of singlet oxygen (Figure 5C and Figure 5E). The molecular beacon is specifically cleavable by $\mathrm{MM} 7$ peptide $\left(\mathrm{PP}_{\mathrm{MMP7}} \mathrm{B}+\mathrm{MMP7}\right.$ in Figure 5C), while uncleavable by $\mathrm{MM} 2$ peptide $\left(\mathrm{PP}_{\mathrm{MMP} 7} \mathrm{~B}+\mathrm{MMP} 2\right.$ in
Figure 5C). Moreover, the addition of MM7 inhibitor $\left(\mathrm{PP}_{\mathrm{MMP} 7} \mathrm{~B}+\mathrm{MMP} 7+\right.$ inhibitor in Figure 5C) cannot restore the fluorescence and singlet oxygen production ability. All these results demonstrate the selectivity of the peptide linker to MM7. The cleavage of the peptide linker was further evidenced by high performance liquid chromatography (HPLC) in Figure 5D. The $\mathrm{PP}_{\mathrm{MMP}} \mathrm{B}$ has a retention time of approximately $26.1 \mathrm{~min}$. The cleavage of $\mathrm{PP}_{\mathrm{MMP7}} \mathrm{B}$ by $\mathrm{MM} 7$ resulted in two fragments peaked at 14.1 and $36.0 \mathrm{~min}$, corresponding to the BHQ3 moiety and Pyro moiety, respectively, which is confirmed by the UV-vis absorption spectroscopy and MALDI-TOF mass spectroscopy. The $\mathrm{PP}_{\mathrm{MMP}} \mathrm{B}$ shows significant photodynamic cytotoxicity (Figure 5F) in KB cells (MMP7 overexpressed cell line) compared with the BT20 cells (MMP7 less expressed cell line). The KB tumors are found to be greatly depressed by the enzyme cleavable molecular beacon $\mathrm{PP}_{\mathrm{MMP} 7} \mathrm{~B}$.

\section{$\mathrm{pH}$}

Liu et al. constructed an imaging-guided $\mathrm{pH}$ sensitive PDT platform by using charge reversible up-conversion nanoparticles (Wang et al., 2013). As shown in Figure 6A, the $\mathrm{pH}$-sensitive polymer PAH-DMMA-PEG (negatively charged) was grafted onto the positive surface of the $\mathrm{Mn}^{2+}$-doped, Ce6 layered up-conversion nanoparticles (UCNPs) via electrostatic interactions (UCNP@2XCe6-DMMA-PEG). At $\mathrm{pH}=6.8$, the nanoparticle zeta potential increasing from approximately $-18 \mathrm{mV}$ to $+10 \mathrm{mV}$, confirming the acid-responsive detaching of the PEG coating from the UCNPs. UCNP@2XCe6-SA-PEG that is not responsive to acid is used as a reference. As expected, 

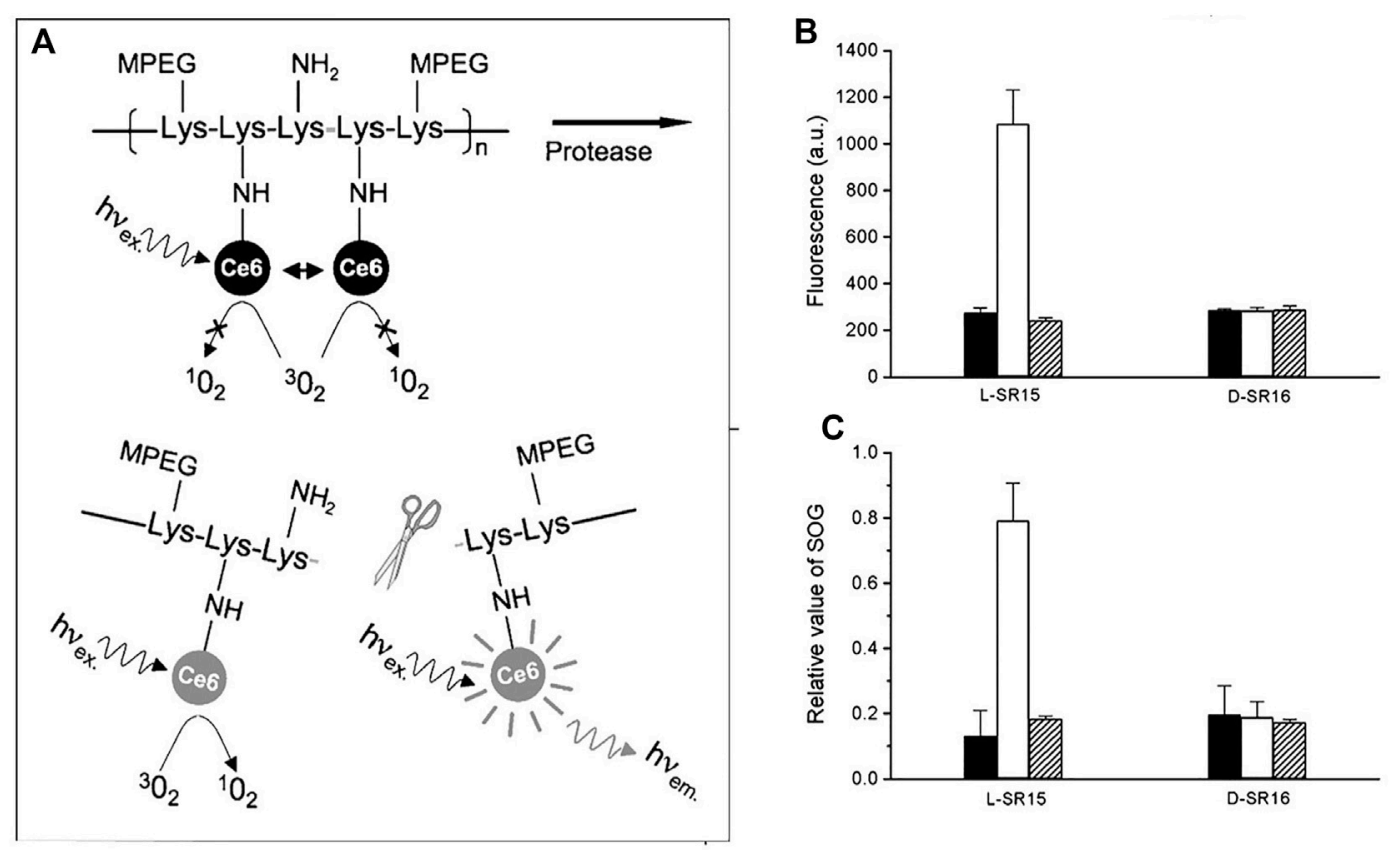

D
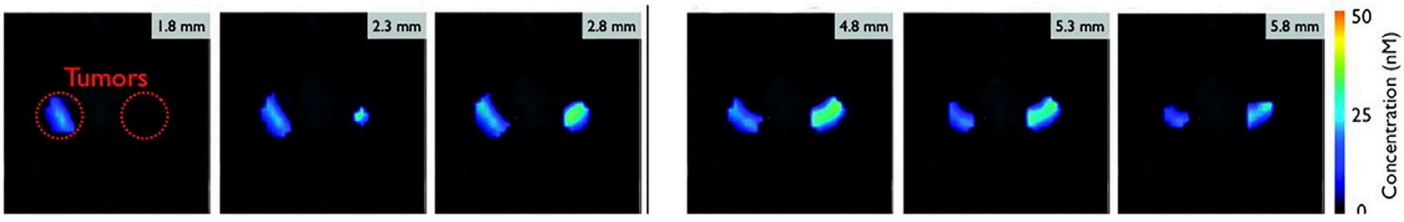

FIGURE 4 | (A) Schematic diagram of PM-PDT strategy; (B) activation of fluorescence intensity and (C) singlet oxygen generation of L-SR15 and D-SR16 with phosphate buffer (black columns), cathepsin B (white columns), and CA-074 inhibitor-pretreated cathepsin B (striped columns); (D) six consecutive slices from a threedimensional fluorescence-mediated tomographic scan (Choi et al., 2006).

the zeta potential remained the same in $\mathrm{pH}=7.4$ and $\mathrm{pH}=6.8$. The ability of the internalization by cancer cells at different $\mathrm{pH}$ was studied by confocal laser scanning microscopy imaging. The UCNP@2XCe6-DMMA-PEG at $\mathrm{pH}=6.8$ shows increased internalization ability than at $\mathrm{pH}=7.4$, hence enhanced phototoxicity to HeLa cells was observed (Figure 6D). Owing to the doping of the $\mathrm{Mn}^{2+}$ to the UCNPs, the materials can be used for dual-modal imaging, i.e., up-conversion luminescence imaging and magnetic resonance imaging (Figure 6B and Figure 6C). Both materials show good accumulation in tumor sites. UCNP@2XCe6-DMMA-PEG has a longer retention in tumor than the reference UCNP@2XCe6-SA-PEG. This is due to the acid-responsive detaching of the PEG coating resulting in a strong positive surface of UCNPs, which enhanced the stickiness of the material to the negatively charged cancer cell membranes and tumor tissues. The longer retention time of UCNP@2XCe6DMMA-PEG contributes to the better PDT efficacy in vivo than UCNP@2XCe6-SA-PEG (Figure 6E).

\section{ROS}

An ROS responsive self-degrading polymer was constructed for enhanced chemotherapy and PDT (Wang et al., 2019). As shown in Figure 7A, the therapeutic prodrug DOX was conjugated to the polymer with a pendant thioketal bond, which is responsive to ROS such as singlet oxygen or hydrogen peroxide. The triplet photosensitizer Ce6 with a planar aromatic structure interacted with the DOX via $\pi-\pi$ stacking, constituting a self-assembled nanoparticle PEG-PBC-TKDOX(Ce6). In the tumor microenvironment or upon light irradiation, the abundant hydrogen peroxide in the tumor microenvironment or the singlet oxygen produced from irradiated Ce6 leads to the cleavage of the pendant thioketal bond, followed by the cascade reaction of self-destructive polymer. Accordingly, the Ce6 and DOX are actively released (Figure 7A). The degradation of the polymer was confirmed by ${ }^{1} \mathrm{H}$-NMR characterization and the decrease of the molecular weight in the presence of hydrogen peroxide. The release of DOX was confirmed by confocal laser scanning microscopy imaging. Free DOX has fluorescence and accumulates in nuclear. As compared with those without light irradiation, KB cells incubated with PEG-PBC-TKDOX(Ce6) upon light irradiation show enhanced accumulation in nuclear, indicating the release of DOX from polymer backbone. Biodistribution of DOX and Ce6 was studied (Figures 7B,C). The light irradiation enhanced the accumulation of the PEGPBC-TKDOX $(\mathrm{Ce}$ 6) in tumor sites. This may be attributed to the ROS induced destruction of the polymer, as smaller molecular 
A
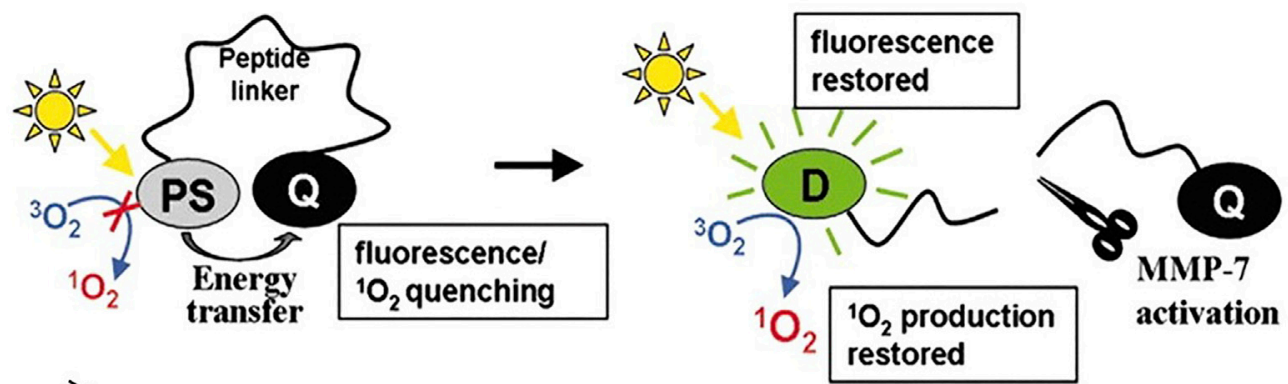

B

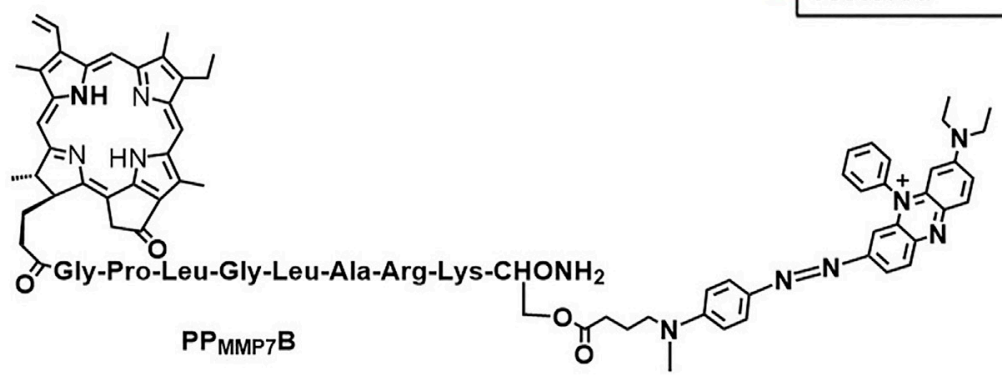

C
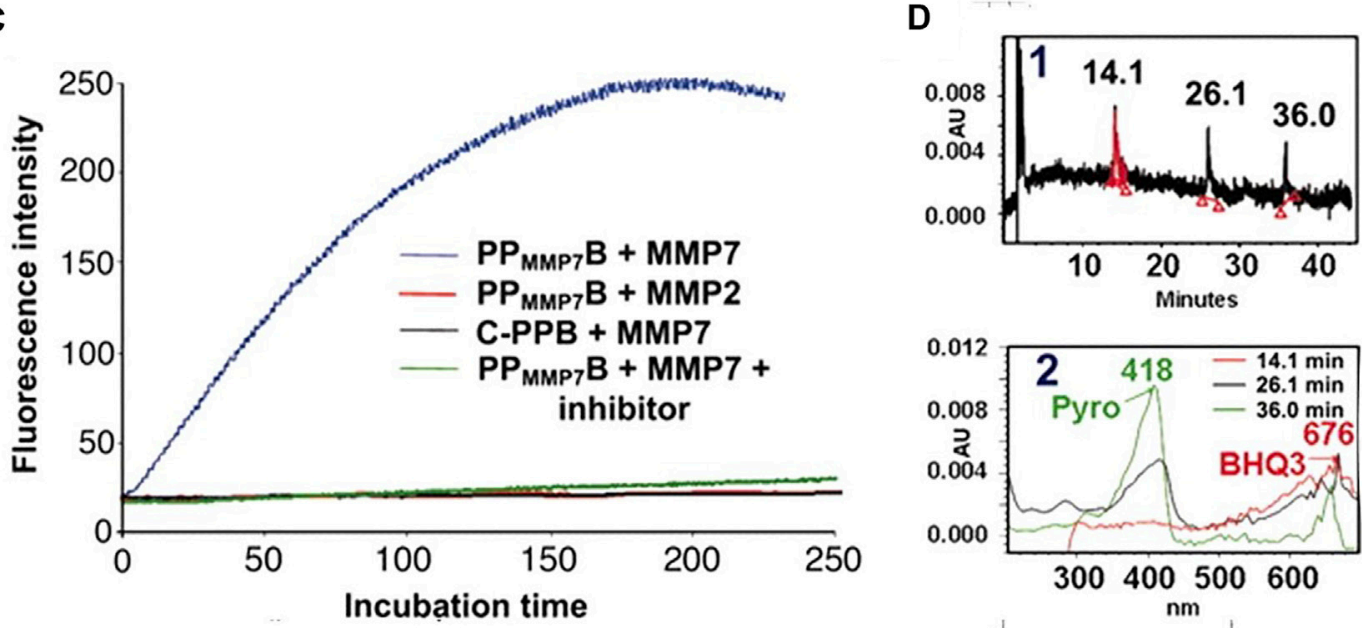

E
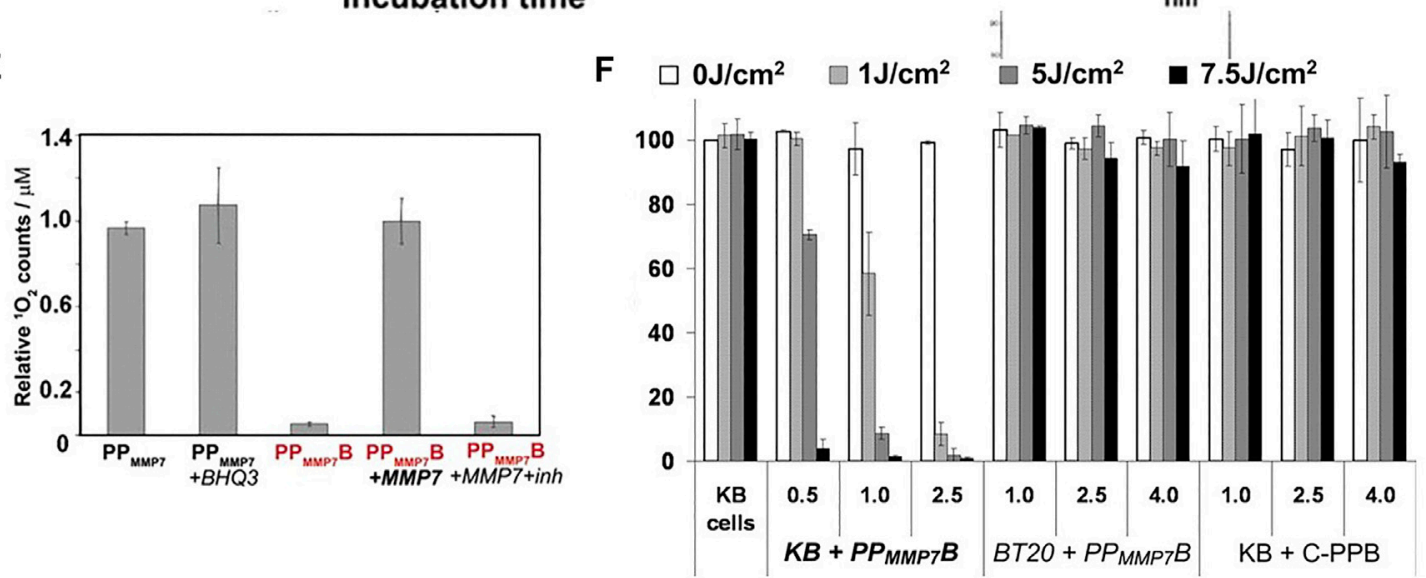

FIGURE 5 | (A) The concept of photodynamic molecular beacons. (B) Molecular structure of PP ${ }_{\text {MMP7 }}$ B. (C) Fluorescence kinetics of different groups. (D) HPLC spectrum of $\mathbf{P P}_{\mathbf{M M P 7}} \mathbf{B}+$ MMP7 incubated at $37^{\circ} \mathrm{C}$ for $2 \mathrm{~h}$ and corresponding UV-vis spectra. (E) The relative ${ }^{1} \mathrm{O}_{2}$ counts of different groups. (F) Photodynamic cytotoxicity determined by MTT assay as a function of PS and light doses, compared with untreated cells. Copyright (2007) National Academy of Sciences (Zheng et al., 2007). 
A
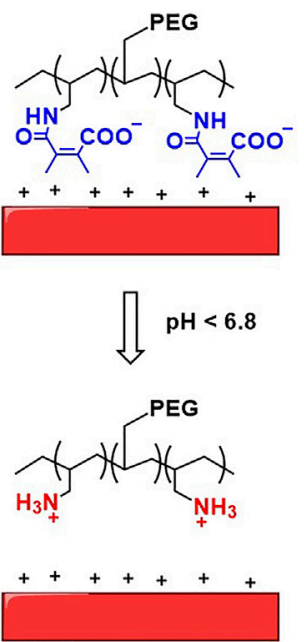

D

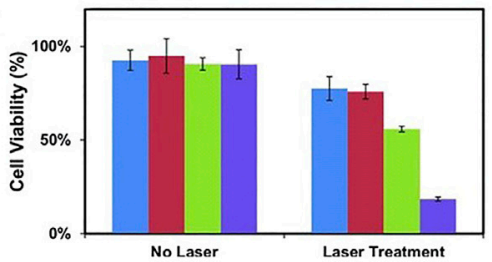

B
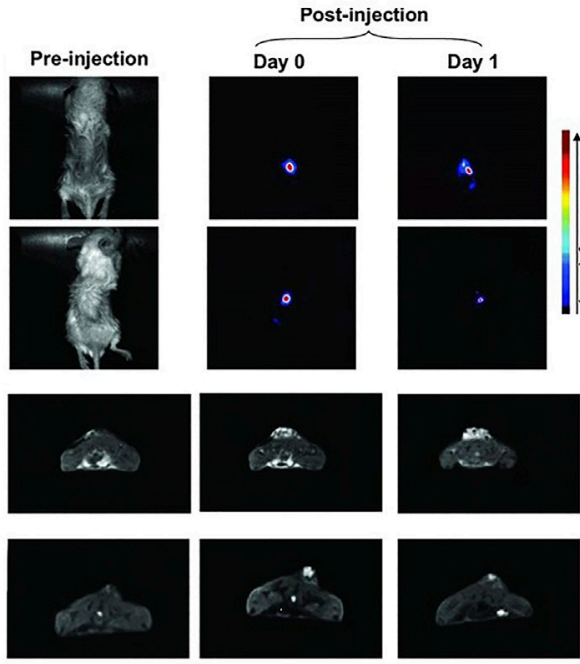

UCNP@2XCe -SA-PEG

E 10

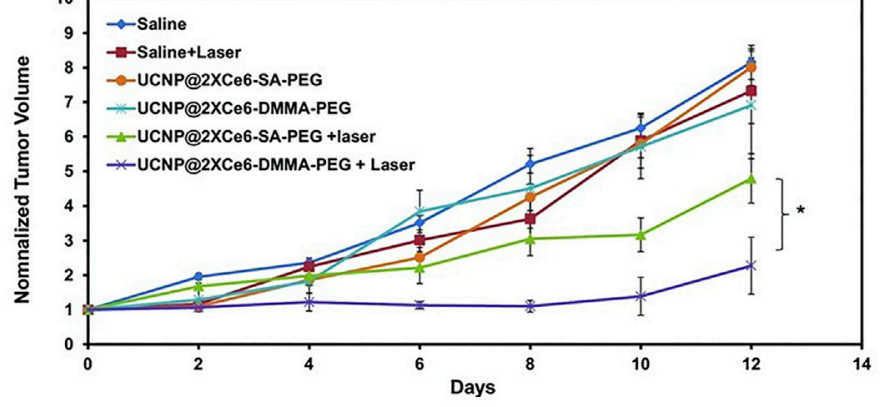

FIGURE 6 | (A) Schematic illustration of pH-responsive smart theranostic UCNPs, showing the detachment of PAH-DMMA-PEG from the positively charged nanoparticle surface under pH 6.8; (B) in vivo UCL images and (C) T 1 MR images of mice after intratumoral injection with UCNP@2xCe6-DMMA-PEG or UCNP@2xCe6-SAPEG. The images were taken at different times post-injection; (D) cell viability data of HeLa cells after various treatments indicated with and without the 980 nm laser irradiation as evaluated by the standard MTT assay; (E) tumor growth curves of different groups of mice after various treatments. Reprinted with permission from Wang et al. (2013).

size is known to have better tumor accumulation ability (Wang et al., 2019). The PEG-PBC-TKDOX(Ce6) upon light irradiation shows outstanding therapeutic efficacy (Figure 7D) and decreased free DOX-related side effects such as decrease of body weight (Figure 7E).

\section{GSH}

Natural polysaccharides have been popularly used for the development of self-quenchable nanoparticles due to their good water solubility, biodegradability, biocompatibility, and tumor targeting ability (Bae et al., 2010; Bae and Na, 2010; Li et al., 2011; Yoon et al., 2012) Kang Moo Huh et al. constructed a GSH responsive pheophorbide a-glycol chitosan system for PDT. As shown in Figure 8C, the photosensitizer pheophorbide a (PheoA) was dangled onto the glycol chitosan via a reducible disulfide bond (PheoA-ss-GC in Figure 8A), which is sensitive to the GSH that is abundant in the tumor microenvironment. The conjugate tends to aggregate into nanoparticles and the fluorescence/singlet oxygen production ability of PheoA is diminished due to self-quenching. The cleavage of the disulfide bond by GSH recovered both the fluorescence and singlet oxygen production ability, achieving the on-site release of the photosensitizer and selectively killing tumor cells. A reference compound (PheoA-GC in Figure 8B) that has no disulfide bond linker was also synthesized for comparison. The recovery of fluorescence and singlet oxygen production ability of PheoA-ss-GC were evidenced both in cuvette and in vitro. As shown in Figure 8D, the fluorescence of PheoA is obvious in the $\mathrm{KB}$ cells treated with PheoA-ss-GC, while it was completely quenched in the cells treated with PheoA-GC. Moreover, the PheoA-ss-GC shows significantly higher phototoxicity to KB cells than PheoA-GC (Figure 8E) as the self-quenching effect of the photosensitizer is greatly alleviated upon the activation of high concentration of GSH in tumor cells. Consequently, outstanding PDT efficacy of PheoA-ss-GC is achieved (Figure 8F).

\section{Hypoxia}

Tumor tissues are known to be hypoxic. Taking advantage of this characteristic, Zhao et al. designed multifunctional micelles that are dually responsive to hypoxia and singlet oxygen ( $\mathrm{Li}$ et al., 2018c). The molecular structure is consisted of azobenzene and imidazole. The hypoxic atmosphere of the tumor tissues induces the collapse of azobenzene and consequently provoked PEG shedding (dePEGylation); the singlet oxygen produced by $\mathrm{Ce} 6$ 


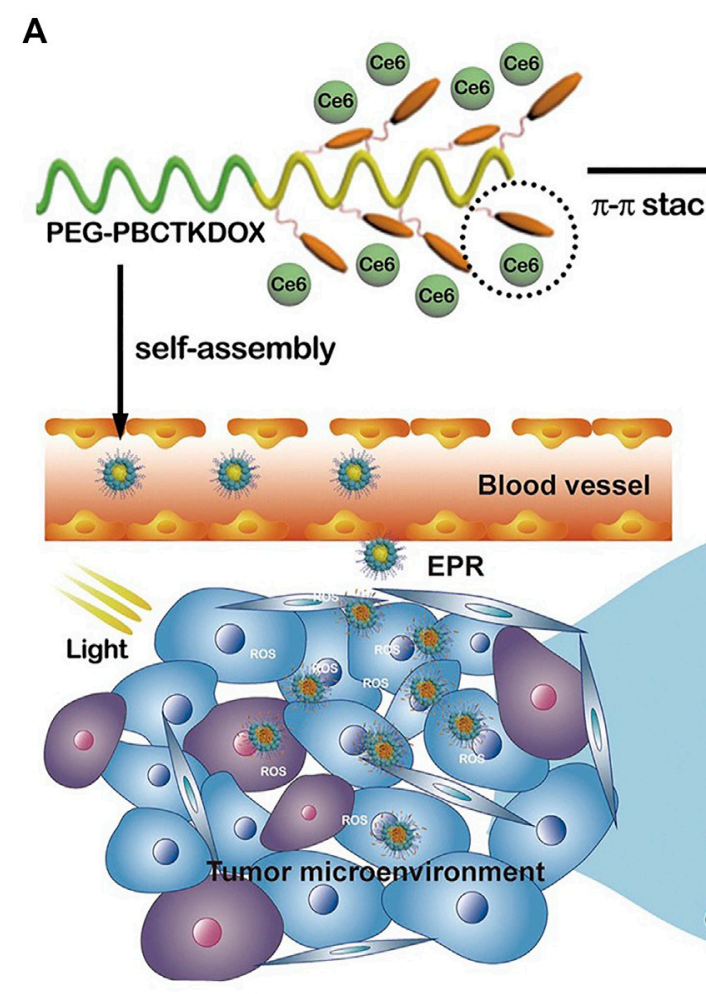

B
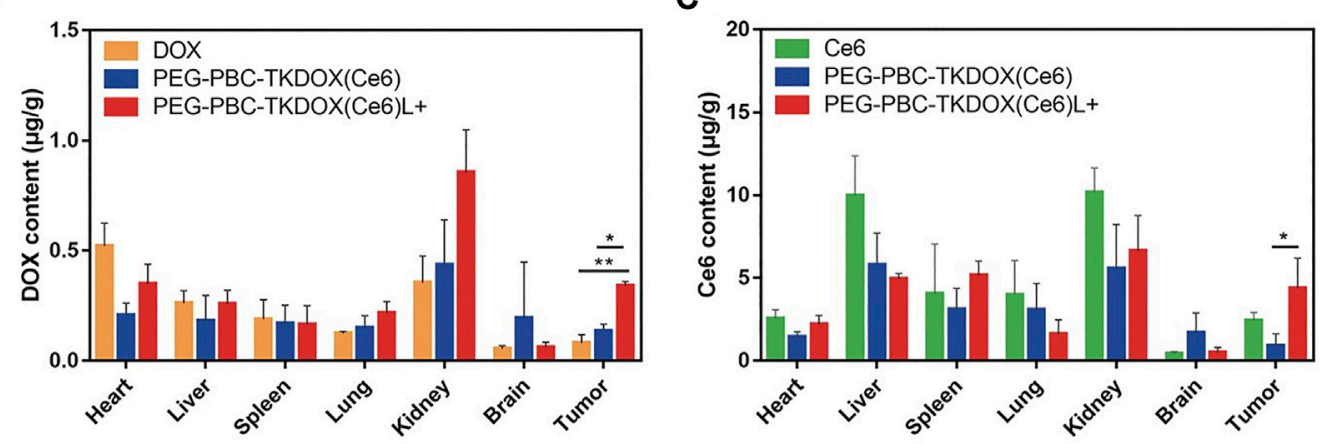

D

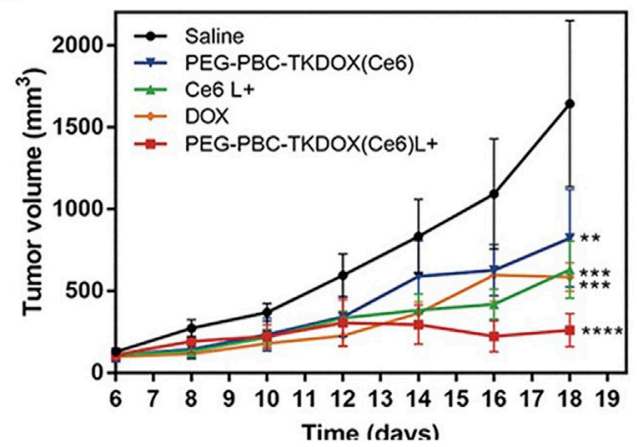

E

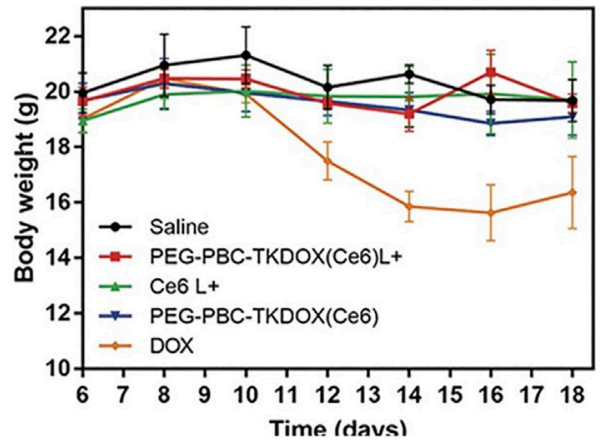

FIGURE 7 | (A) Schematic drawing of the cascade reaction of self-destructive polymeric nanomicelles; (B) biodistribution of DOX solution and PEG-PBC-TKDOX (Ce6) micelles at $12 \mathrm{~h}$ with or without light irradiation; (C) biodistribution of Ce6 solution and PEG-PBC-TKDOX (Ce6) micelles at $12 \mathrm{~h}$ with or without light irradiation; (D) growth curves of subcutaneously inoculated KB tumors. Intravenous injections were given to mice at day 7, day 9, and day 11 . The $660 \mathrm{~nm}$ laser irradiation followed at $12 \mathrm{~h}$ after each injection; (E) body weight changes $\left(n=5,{ }^{\star \star}, p<0.01,{ }^{\star \star *}, p<0.001,{ }^{\star \star * \star *}, p<0.0001\right)$. Reprinted with permission from Wang et al. (2019). 

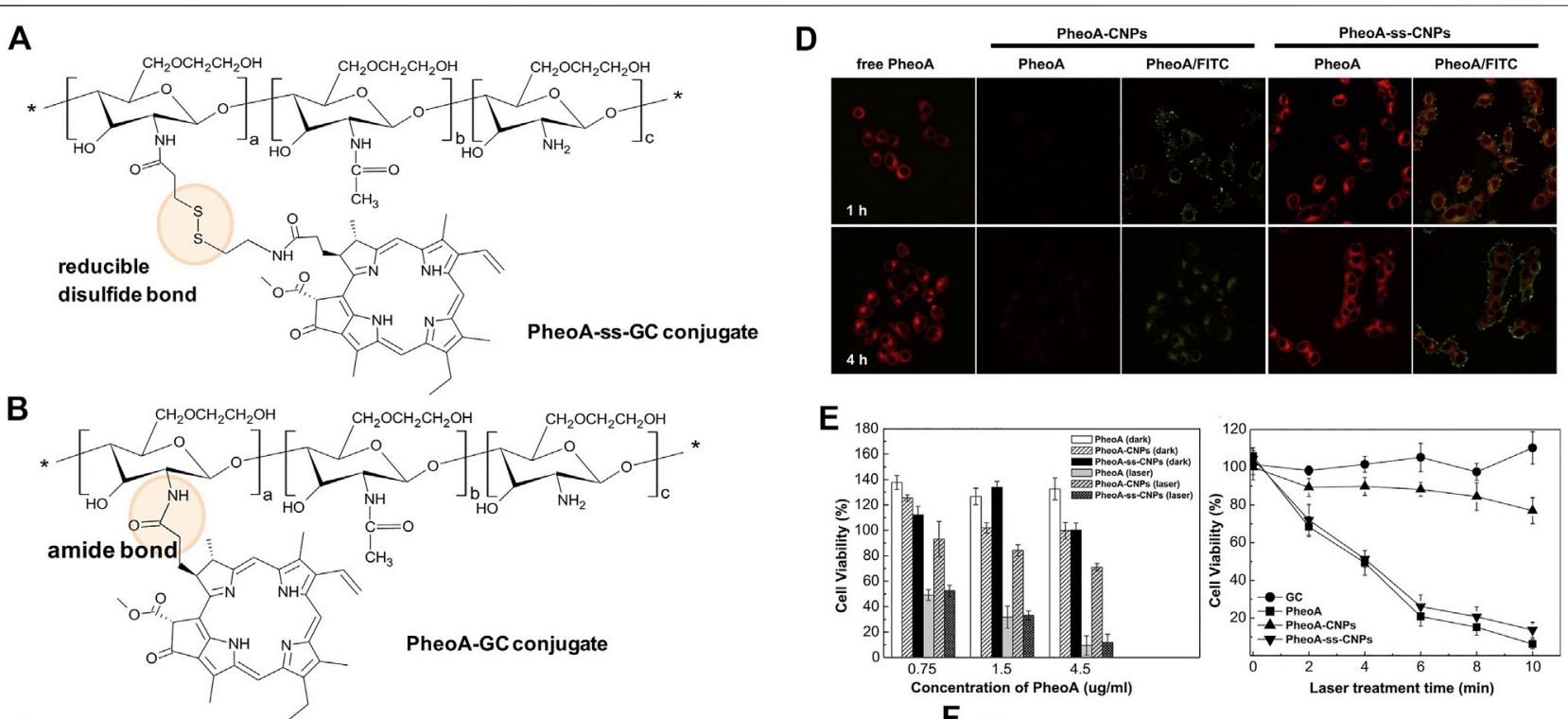

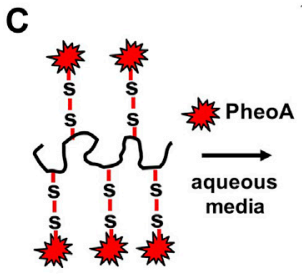

PheoA-ss-GC conjugate

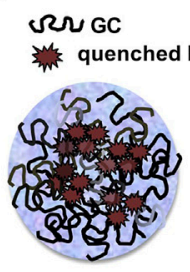

PheoA-ss-CNPs in self-quenched state

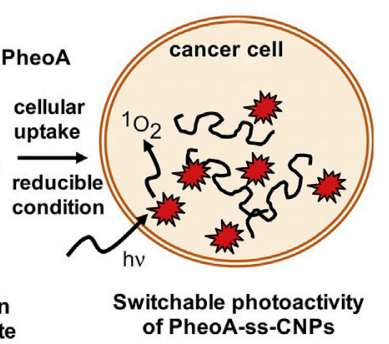

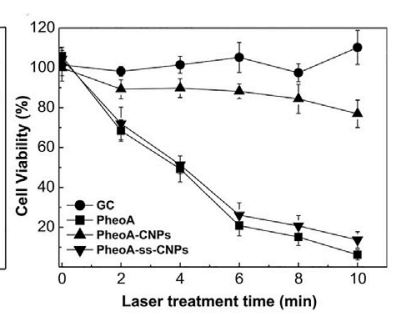

$F$

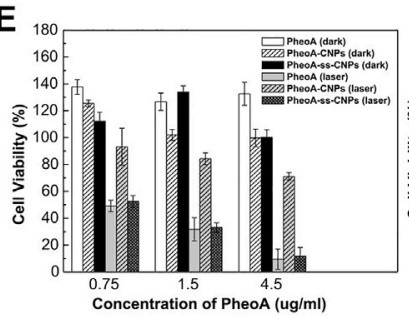

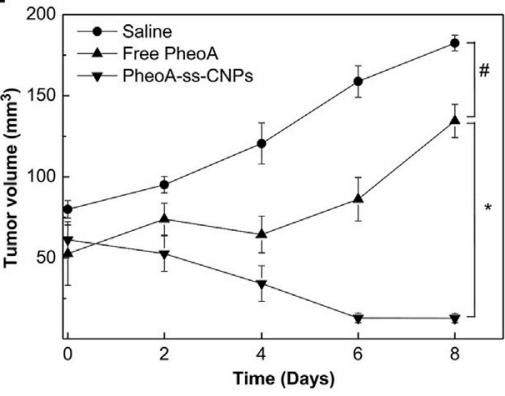

FIGURE 8 | Chemical structures of (A) PheoA-ss-GC and (B) PheoAeGC conjugates and (C) illustration of bioreducible PheoA-ss-CNPs for switchable photoactivity of PheoA; (D) CLMS images of KB cells treated with free PheoA, PheoAeCNPs, and PheoA-ss-CNPs; (E) phototoxicity and dark toxicity of free PheoA, PheoAeCNPs, and PheoA-ss-CNPs; (F) tumor growth of HT-29 tumor-bearing mice after various treatments. Reprinted with permission from Oh et al. (2013).

upon light irradiation leads to an oxidation of hydrophobic imidazole to hydrophilic urea, followed by a rapid release of Ce6. Enhanced internalization of micelles by LLC cells and improved PDT efficacy is observed.

\section{Temperature}

Besides the internal triggers such as $\mathrm{pH}$, enzyme, GSH, and hypoxia, external triggers such as temperature can also activate the PDT. Poly (N-isopropylacrylamide) (PNIPAM) is a frequently used thermo-sensitive polymer (Liu et al., 2009; Lv et al., 2015). It can go through the phase transition from hydrophilic to hydrophobic state when the temperature is above the lower critical solution temperature (LCST) (Stöber et al., 1968; Pelton and Chibante, 1986; Pelton, 2000). The LCST of PNIPAM in water is approximately $32-33^{\circ} \mathrm{C}$. Molecular structure modification to the PNIPAM (co-polymerization with other monomers) or adjusting the $\mathrm{pH}$ value can affect this LCST value (Hoare and Pelton, 2004; Rijcken et al., 2007; Dai et al., 2012; Zhang et al., 2013; Jiang et al., 2014). Hence it is possible to adjust this LCST to suitable values such as body temperature $\left(\mathrm{ca} .37^{\circ} \mathrm{C}\right)$. In addition, tumor tissues are reported to have a higher temperature than normal tissues due to inflammation and cancer cell immortalization (Wang et al., 2013; Lv et al., 2015)

A nanoplatform based on the $\mathrm{pH}$-temperature sensitive polymer was constructed for cancer therapy (Xu et al., 2016). As shown in Figure 9, the up-conversion nanoparticles (UCNP) are decorated with carbon dots and chemotherapeutic reagent DOX and PDT reagent zinc(II)phthalocyanine $(\mathrm{ZnPc})$. The up-converted fluorescence excited the $\mathrm{ZnPc}$ to its triplet state and produce singlet oxygen, and the decorated carbon dots generate photothermal effect upon near infrared laser irradiation. This thermal effect at tumor acidic tissues induces the shrinkage of $\mathrm{P}$ (NIPAm-MAA) polymer, leading to the controlled drug release. As shown in Figure 9F, the nanoplatform shows significant drug release at $50^{\circ} \mathrm{C}(\mathrm{pH}=$ 7.4). Moreover, the drug can be efficiently released at acidic conditions $(\mathrm{pH}=4.0$, Figure $9 \mathrm{G})$ at $37^{\circ} \mathrm{C}$. These results show that the drug release can be controlled by $\mathrm{pH}$ and temperature. The thermal effect of the nanoplatform upon NIR laser irradiation was shown in Figure 9D. The NIR laser irradiation to the material accumulated in tumor site increased the local temperature, hence the drug release 

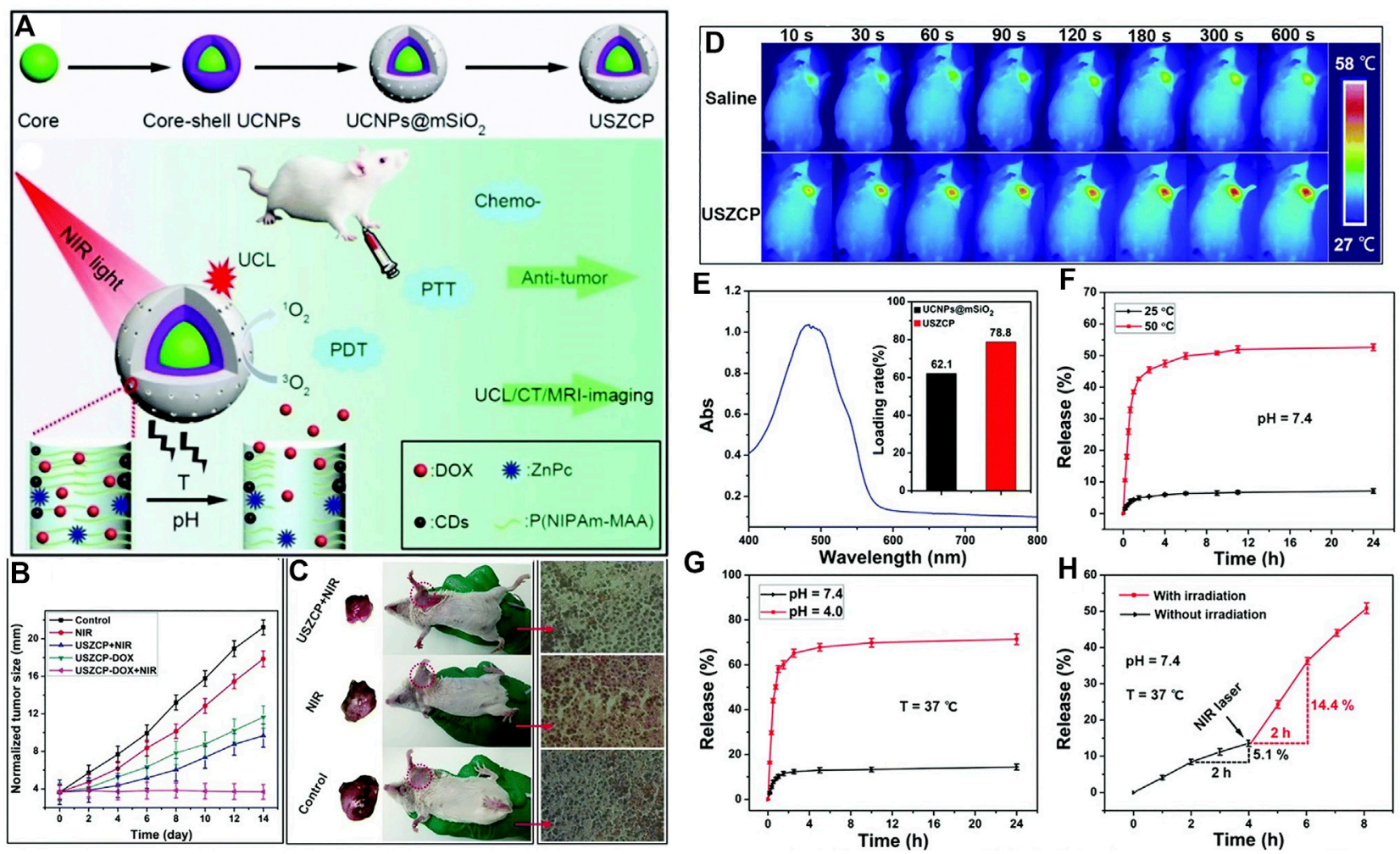

FIGURE 9 | (A) Schematic illustration of $\mathrm{pH}$-temperature sensitive polymer for cancer therapy. (B) Changes in the tumor size of the $\mathrm{H} 22$ tumor obtained from mice after different treatments; (C) Picture of tumor and H\&E staining; (D) in vivo infrared thermal images of a tumor-bearing mouse after injection of USZCP under NIR irradiation with different times; (E) UV-vis absorption spectrum of DOX (inset shows the DOX loading content of UCNPs@mSiO2 and USZCP); the release efficiency of USZCP-DOX at different (F) temperatures and (G) pH values; (H) the release efficiency of USZCP-DOX triggered by a NIR laser. Reprinted with permission from Xu et al. (2016).

upon NIR laser irradiation can be expected (Figure 9H). The combination of the PDT and chemotherapy shows an outstanding therapeutic efficacy (Figure 9B and Figure 9C).

\section{SMART POLYMERIC DELIVERY SYSTEM FOR ANTIMICROBIAL PHOTODYNAMIC THERAPY}

Antibiotics have been the most effective strategies to fight against bacterial infections. However, the abuse of antibiotics has led to severe antimicrobial resistance (Bush et al., 2011). There are approximately 700,000 deaths per year due to antimicrobial resistance all over the world (Klausen et al., 2020). Developing other anti-bacterial strategies such as PDT that are alternatives to antibiotics is of great urgency (Demidova and Hamblin, 2004; Hamblin and Hasan, 2004; Pagonis et al., 2010; Huang et al., 2012).

The delivery of the photosensitizer to the bacteria (especially Gram-negative bacteria) is not a trial task due to its structural features. As shown in Figure 10, the cell wall of Gram-positive bacteria consists of thick, porous layers of peptidoglycan embedded with proteins and lipoteichoic acid. The porous layers are relatively easy to go through for photosensitizers. Moreover, the lipoteichoic acids on the outside are negatively charged, which tends to bind with cationic agents (Minnock et al., 2000; Lambert, 2002). As a comparison, the cell wall of Gramnegative bacteria is thicker, being composed of a peptidoglycan layer, the inner cytoplasmatic membrane, and the outer membrane. This additional outer membrane of Gram-negative bacteria is composed of phospholipids and lipopolysaccharides, which is an effective barrier that limits the penetration of photosensitizers (Minnock et al., 2000). Hence, a smart polymeric delivery system that can target the bacteria and selectively kill the bacteria is necessary for optimal antimicrobial PDT.

\section{Bacterial-Target Polymeric Delivery System Structure-Inherent Targeting}

As it has been mentioned that the structures of the tumor cells and the bacteria are different, photosensitizers that are commonly used in PDT of tumors cannot always be fit for the PDT of bacteria, especially Gram-negative bacteria (Malik et al., 1992). For instance, the cell wall of Gram-negative bacteria is thick and it has been a tough issue to deliver the photosensitizer into the Gram-negative bacteria. Hence, the Gram-negative bacteria are notoriously difficult to deal with (Malik et al., 1992).

As the bacteria are heavily negatively charged, the cationic photosensitizers are beneficial for their penetration and targeting to bacteria via electrostatic interactions (Minnock et al., 1996; 

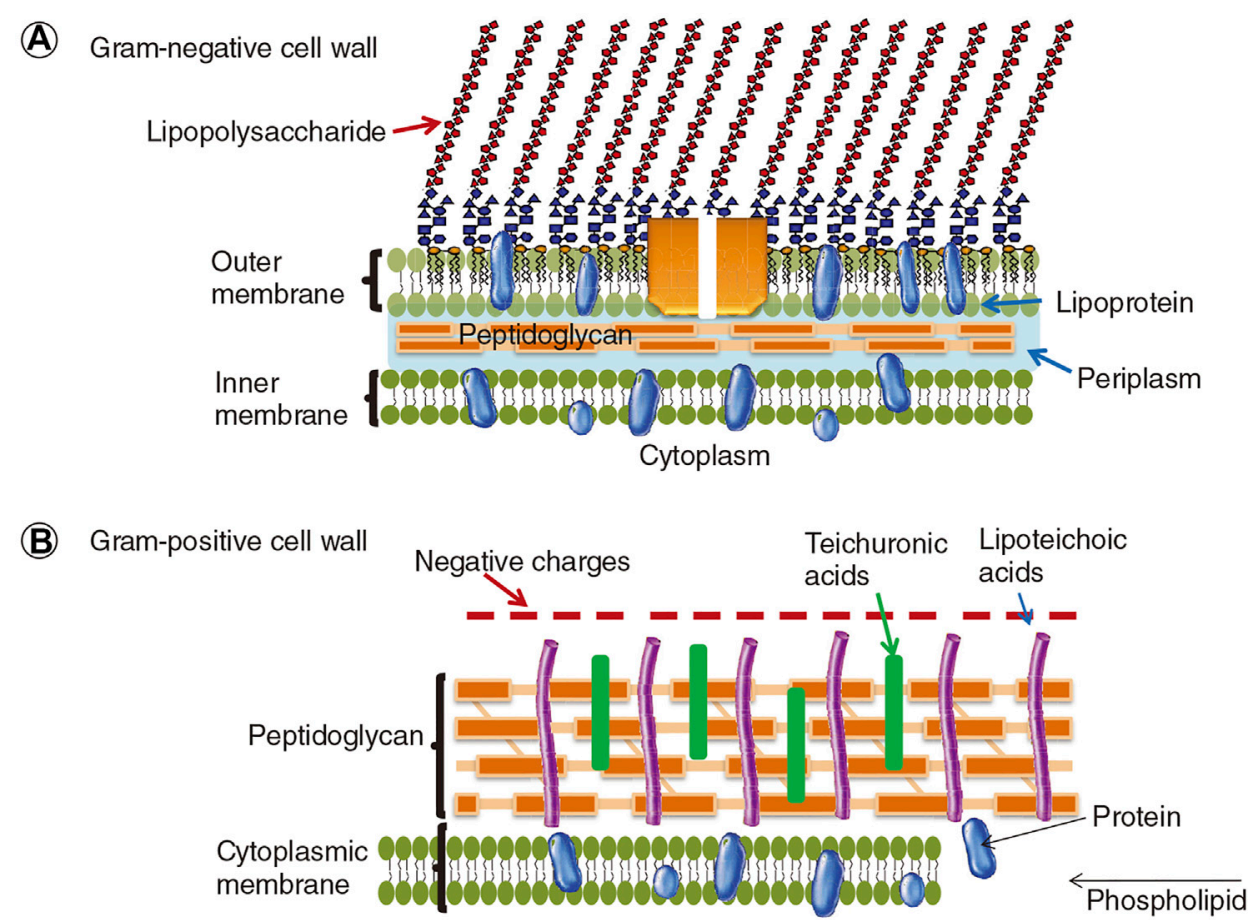

FIGURE 10 | The cell walls of Gram-negative and Gram-positive bacteria. Reprinted with permission from Yin et al. (2015).

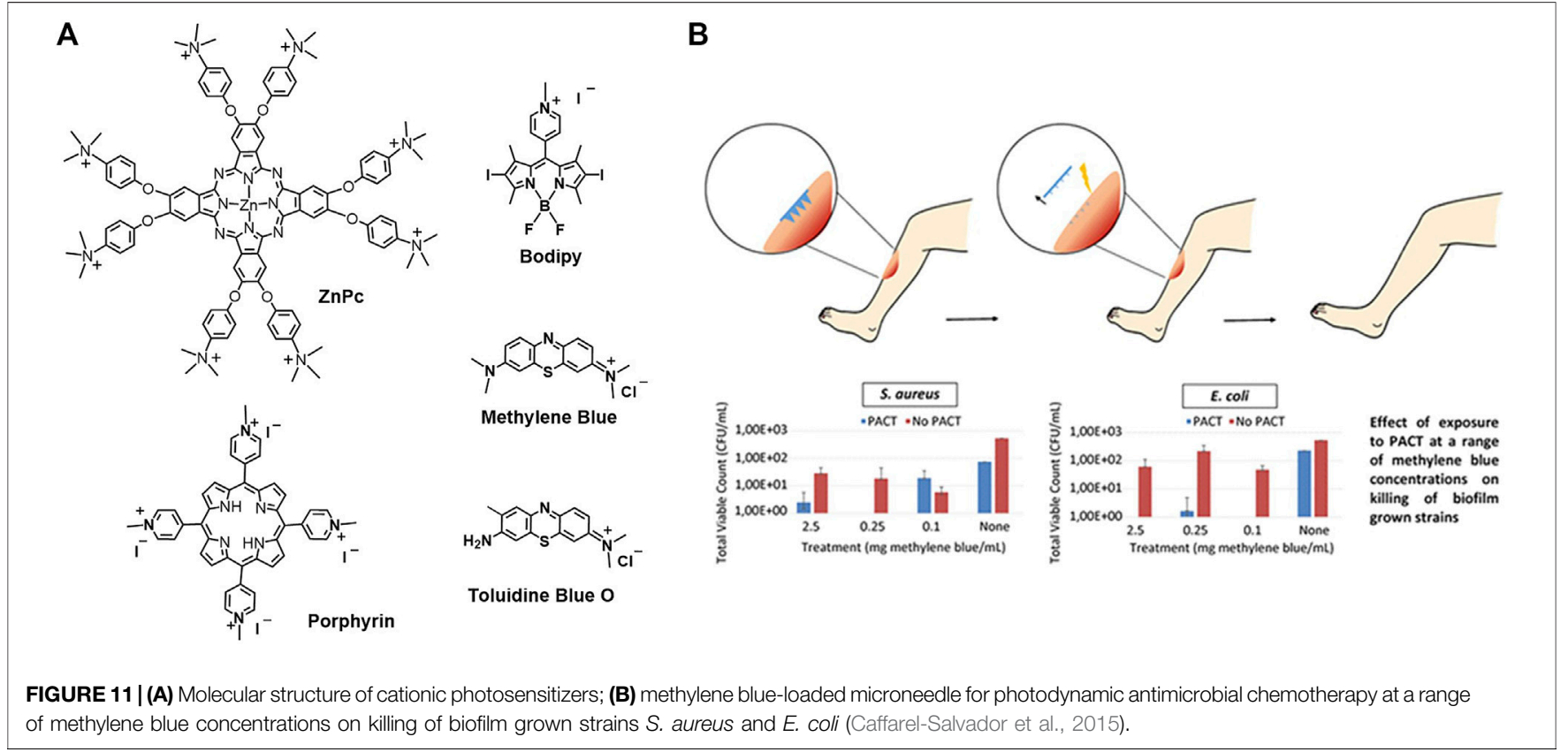

Chen et al., 2020). This is a kind of structure-inherent targeting. Cationic photosensitizers such as methylene blue, cationic porphyrins, cationic Bodipy, and cationic phenoxyphthalocyaninato zinc (II) are shown as examples in Figure 11A.

\section{Active Targeting}

Similar to the anti-tumor therapy, there is also active targeting strategy to achieve better PDT efficacy. As the structures of tumor cells and bacteria are different, the target point also varies. For instance, the anti-tumor therapy targets the tumor vasculature, 
tumor cells, and tumor subcellular organelles such as mitochondria, lysosome, nucleus, and endoplasmic reticulum. While the anti-bacterial therapy targets the bacteria membranes, bacterial exopolysaccharides (EPS), fimbriae, etc. For instance, various EPS are produced by bacteria to create a binding network between pathogens. Hence, bacterial EPS extracted from Lactobacillus plantarum can be utilized as targeting moiety ( $\mathrm{Li}$ C. et al., 2018; Klausen et al., 2020). In addition, glycan is a commonly used targeting moiety with specific binding to lectins. Concerning to this aspect, different sugars such as mannose, sialic acid, and galactose have been utilized to target different pathogens. For instance, D-mannose has been used to bind to FimA protein of Escherichia coli (E. coli) (Krogfelt et al., 1990). As a deeper investigation and understanding to the bacteria, more and more target moieties are emerging.

\section{Physical Penetration Targeting}

As it has been introduced that the bacteria, especially Gramnegative bacteria, have thick cell walls, it makes them very difficult to deal with. Physical penetration by microneedles is an attractive strategy to go across the barriers and target the bacteria (Henry et al., 1998; Lee et al., 2008; Tuan-Mahmood et al., 2013; Donnelly et al., 2014; Kearney et al., 2014; Park et al., 2014). Methylene blue-loaded dissolving microneedles were constructed for antimicrobial PDT (Caffarel-Salvador et al., 2015). The microneedles were fabricated with aqueous blends of Gantrez ${ }^{\circledR}$ AN-139 co-polymer loading with different percentages of methylene blue. As presented in Figure 11B, the microneedles are in the micron range and are arranged in arrays. Loading high percentages of methylene blue $(5 \% \mathrm{w} / \mathrm{w})$ resulted in a decrease of needle height and microneedle strength. Loading suitable percentages of methylene blue $(0.5 \% \mathrm{w} / \mathrm{w})$ shows good mechanical strength that enables the insertion of the microneedles into the wound. The insertion distance is in the range of $378-504 \mu \mathrm{m}$, which is measured with a human tissue mimicker Parafilm ${ }^{\mathrm{TM}}$. The microneedles are expected to dissolve, followed by the release of the methylene blue. Upon photoirradiation, efficient bactericidal activity against Staphylococcus aureus (S. aureus) and E. coli biofilms was observed (Figure 11B).

\section{Bacterial-Activatable Polymeric Delivery System}

Due to the encapsulation of EPS, the biofilm microenvironment is lack of oxygen, which leads to anaerobic glycolysis (Flemming et al., 2016). Recalling from the tumor-activatable polymeric delivery system section, the tumor tissues are also hypoxic. The anaerobic glycolysis or the anaerobic respiration contributes to the acidic and highly reductive microenvironment of both bacterial biofilms and tumor tissues (Gales et al., 2008; Chen et al., 2013; Koo et al., 2017). For instance, the $\mathrm{pH}$ in the methicillin resistant Staphylococcus aureus (MRSA) biofilm microenvironment is less than 5.5, and the concentration of GSH in E. coli biofilm is up to $10 \mathrm{mM}$ (Fux et al., 2005; Gales et al., 2008; Chen et al., 2013; Klare et al., 2016). Hence, the microenvironment of bacteria is similar to that of tumor, which is hypoxic, acidic, highly reductive, and has abundant ROS. Taking advantage of the differences between bacteria and normal tissues, the activatable polymeric delivery system can be developed, achieving selectively killing of the bacteria while keeping the host normal tissue unaffected. Hence, decreased side effect is expected. Herein we presented some typical examples of activatable polymeric delivery systems for anti-bacteria PDT (Radovic-Moreno et al., 2012; Li F. et al., 2018; Zhao et al., 2019; Broenstrup et al., 2021; Wu et al., 2021; Ye et al., 2021).

\section{Enzyme}

Hypocrellin A-loaded lipase sensitive polymer system was reported. The Hypocrellin A is a perylenequinoid pigment that is isolated from the tradition Chinese medicine and it is reported to have singlet oxygen production ability, hence it functioned as a photosensitizer. The lipase sensitive polymer methoxy poly (ethylene glycol)-blockpoly ( $\varepsilon$-caprolactone) (mPEG-PCL) selfassembled to micelles, which is used to encapsulate the photosensitizer Hypocrellin A and enhance its water solubility. As shown in Figure 12A, this Hypocrellin A-loaded polymer micelles can be degraded by the bacterial lipase, along with the release of the photosensitizer. The release of Hypocrellin A in the presence of lipase in PBS was confirmed and presented in Figure 12C. An in vitro antibacterial study shows that the light irradiation significantly reduced the minimum inhibitory concentration (MIC) and minimum bactericidal concentration (MBC) values ( 0.69 and $1.38 \mathrm{mg} / \mathrm{L}$, respectively), as compared with that in the dark (250 and $500 \mathrm{mg} / \mathrm{L}$, respectively). This result demonstrated that the antibacterial activity is mainly attributed to PDT. An in vivo antibacterial study shows that the MRSA in spleen and blood is cleared up and the bacteria in liver is significantly reduced upon PDT (Figure 12D). Compared with the free Hypocrellin A, the polymeric micelles show slightly higher MIC and MBC values, which might be due to the incomplete release of Hypocrellin A. However, the lipase sensitive polymeric micelles achieve significantly increased survival rate, indicating that the lipase sensitive polymeric micelles are a potent polymeric system for combating MRSA infection.

\section{$\mathrm{pH}$}

A pH-sensitive, surface charge switchable supramolecular polymeric system was constructed ( $\mathrm{Hu}$ et al., 2019). As shown in Figure 13A, the $\mathrm{pH}$-sensitive poly (ethylene glycol) (PEG) block polypeptide copolymer [PEG-(KLAKLAK)2-DA] interacted with the $a-C D$ prodrugs (PDT therapeutics $a-C D$ Ce6 and NO therapeutics $\alpha$-CD-NO) via host-guest interaction, forming a negatively charged supramolecular nanocarrier at physiological $\mathrm{pH}$. The negative charge is in favor of the longterm blood circulation. While in acidic conditions $(\mathrm{pH}=5.5)$, the amide bond tends to be cleaved, leading to a switch of the surface charge of the nanocarrier. The obtained positive charge is beneficial for the penetration into biofilms due to the stronger interaction and adhesion to the negatively charged bacterial membrane. As compared with the surface charge unswitchable nanocarrier a-CD-Ce6-NO-SA, the surface charge switchable 
A

MPEG- $\boldsymbol{b}$-PCL

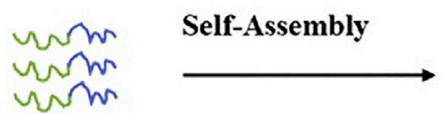

mPEG MW= $10000 \mathrm{Da}$, PCL MW= $5000 \mathrm{Da}$
mPEG-PCL Micelle

mPEG-PCL/HA Micelle
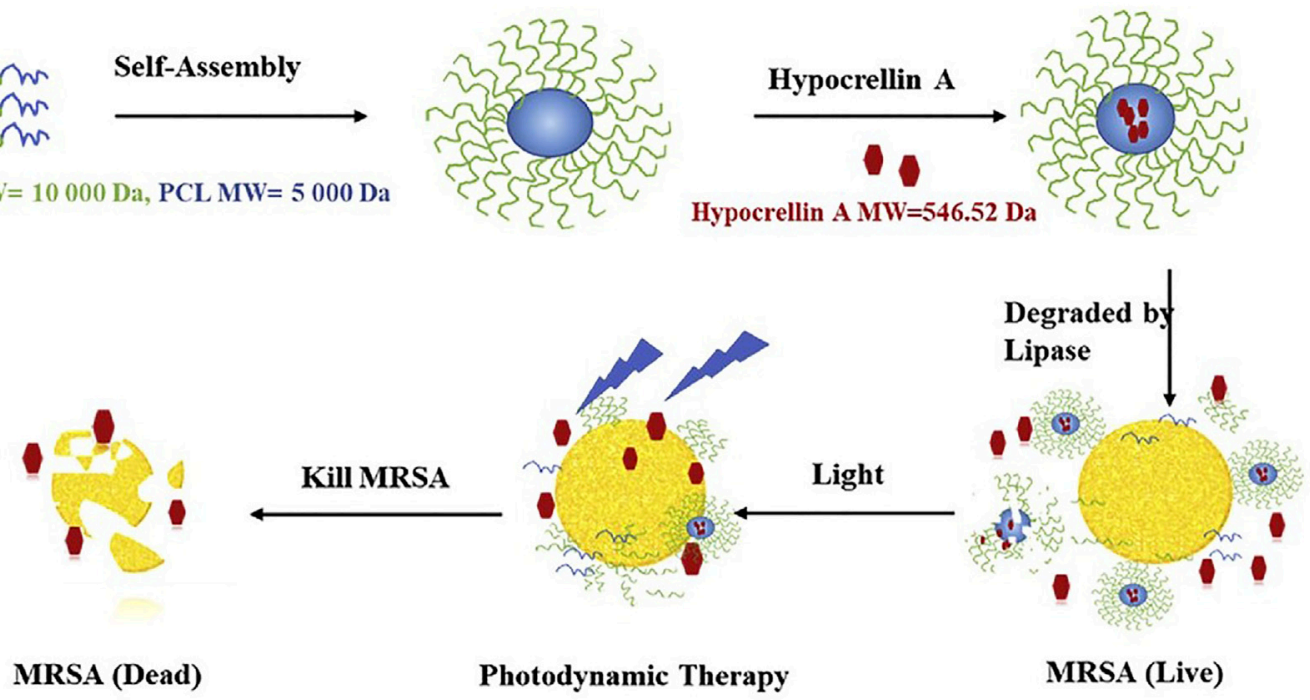

B

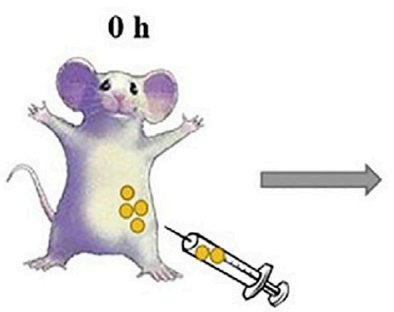

Acute peritoneal infection
2 h

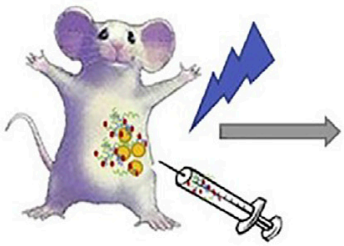

Injection of mPEGPCL/HA micelles

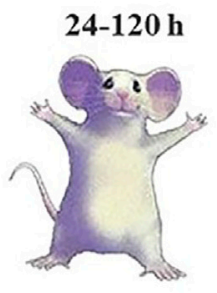

Evaluation of treatment
Tissue samples

Blood samples
C

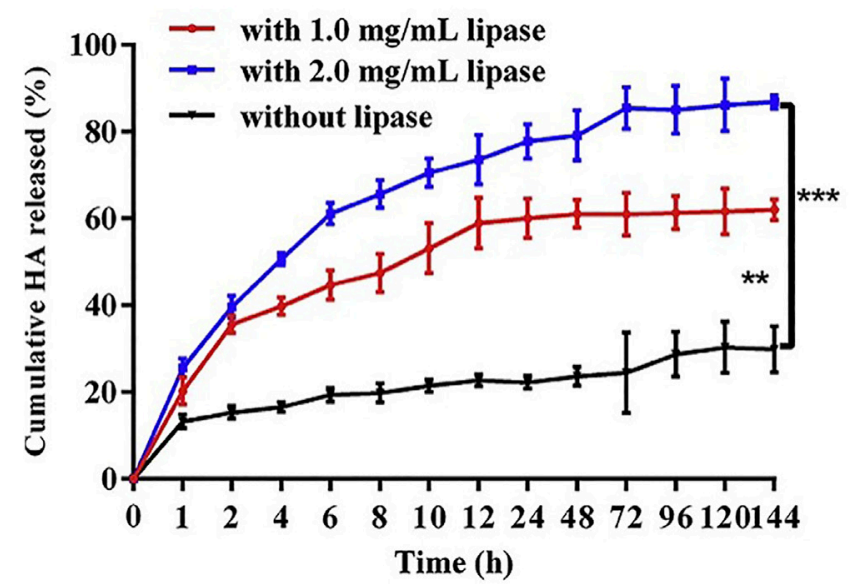

D

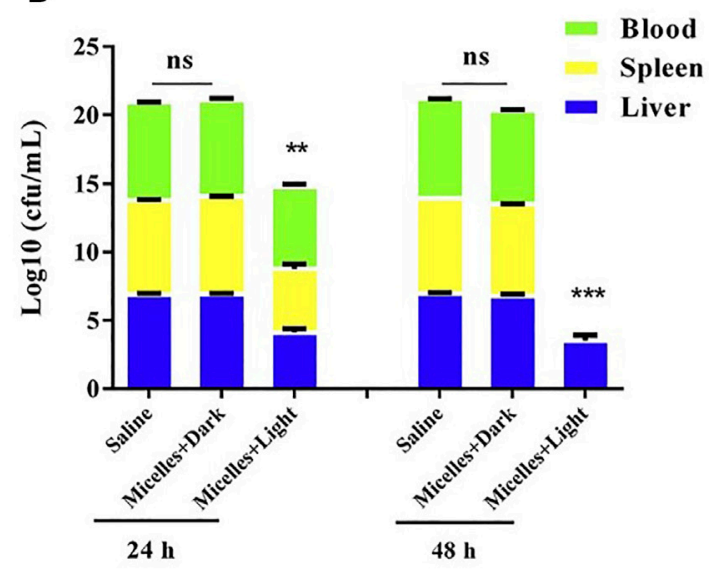

FIGURE 12 | (A) Schematic illustration of the mPEG-PCL/HA micelles for enhanced photodynamic antibacterial activity; (B) schematic illustration of the mPEG$\mathrm{PCL} / \mathrm{HA}$ micelles for the in vivo treatment; (C) cumulative release of HA from micelles without or with lipase $(1.0,2.0 \mathrm{mg} / \mathrm{mll})$; (D) total bacterial counts in the liver, spleen, and blood of mice after being treated with mPEG-PCL/HA micelles (HA dose: $5 \mathrm{mg} / \mathrm{kg}$ ) in dark or light irradiation for 24 and 48 h. Reprinted with permission from Guo et al. (2020). 

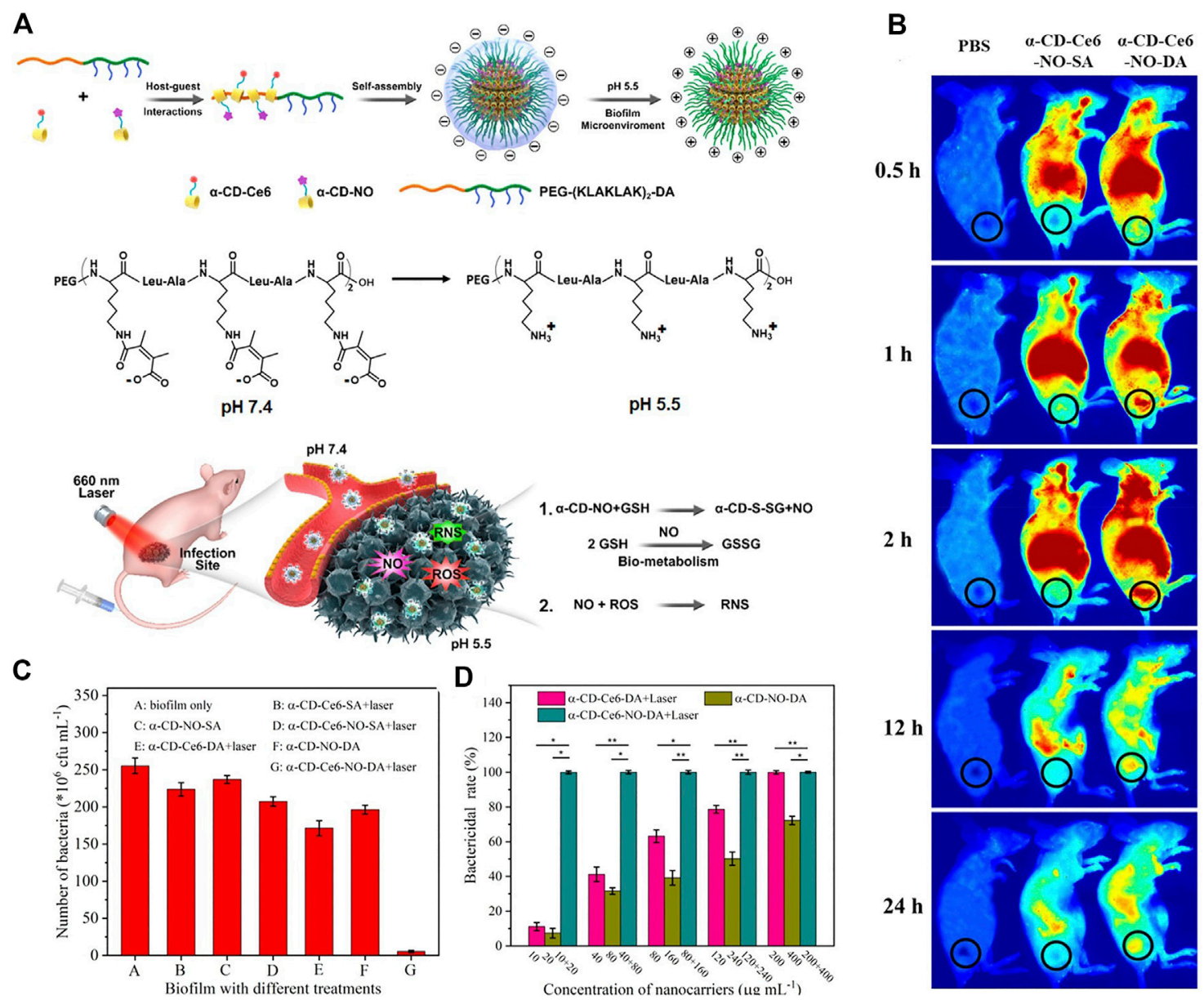

FIGURE 13 | (A) Schematic diagram of the acid-activated charge reversal of PEG-(KLAKLAK)2-DA at pH 5.5 and the mechanisms of the MRSA biofilm associated infection eradication by synergistic effects between ROS and NO produced by a-CD-Ce6-NO-DA nanocarriers; (B) in vivo time-dependent body fluorescence imaging of the MRSA biofilm infected mice after various treatments; (C) related bactericidal results of different nanocarriers with the same Ce6 concentration under laser irradiation, characterized by the standard plate counting assay. (D) In vivo bactericidal rates of various treatments. Reprinted with permission from Hu et al. (2019).

nanocarrier $a-C D-C e 6-N O-D A$ shows enhanced penetration to biofilms and accumulation in MRSA biofilm infected area (Figure 13B). Hence, it is reasonable that upon light irradiation, enhanced bactericidal rate was observed for $a-C D$ Ce6-NO-DA as compared with a-CD-Ce6-NO-SA (Figure 13C and Figure 13D).

Another $\mathrm{pH}$-sensitive charge reversible nanoparticle system was constructed for enhanced penetration and antibacterial PDT efficiency (Wu et al., 2021). As shown in Figure 14A, the photosensitizer Rose Bengal is dangled with dopamine, and then decorated with polymyxin $\mathrm{B}$ (PMB) and gluconic acid (GA) layer-by-layer. Resembling the isoelectric point of protein, the resulted nanoparticles (RB@PMB@GA) are negatively charged at physiological $\mathrm{pH}$ while they switched to positive charged when the $\mathrm{pH}$ is below 5.5, due to the presence of an amino group and a carboxyl group. Consequently, the antibacterial efficiency is greatly improved at $\mathrm{pH}=5.0$ (Figure 14B), due to the $\mathrm{pH}$-sensitive charge inversion. To be specific, as presented in Figure 14C, only Gram-positive bacteria S. aureus were effectively killed upon light irradiation at $\mathrm{pH}=7.4$, while the Gram-negative bacteria $E$. coli were not affected unless in acidic condition $(\mathrm{pH}=5.0)$. This indicates that the charge inversion in acidic condition enhanced the penetration and adhesion to bacteria. As the positively charged polymers are expected to quickly infiltrate biofilms via electrostatic interactions, the nanoparticles show outstanding biofilm penetration and eradication ability (Figures 14D-F).

\section{ROS}

A $\mathrm{pH} / \mathrm{H}_{2} \mathrm{O}_{2}$ dual responsive polymeric system to combat the $S$. aureus and its biofilm is presented in Figure $15 \mathbf{A}$ (Zhao et al., 2021) The polymer POEGMA-b-PBMA is assembled with a surface charge-switchable photosensitizer, 5,10,15,20-tetra- $\{4$ [3-(N,N-dimethyl-ammonio) propoxy]phenyl porphyrin (TAPP) into nanoparticles with an average diameter of $180 \mathrm{~nm}$. The large amount of $\mathrm{H}_{2} \mathrm{O}_{2}$ in $\mathrm{S}$. aureus reacts with the arylboronic ester moiety and induces the disintegration of the nanoparticles, followed by the release of TAPP. The TAPP is further protonated in an acidic bacterial microenvironment, which increases its hydrophilicity and reduces its self- 

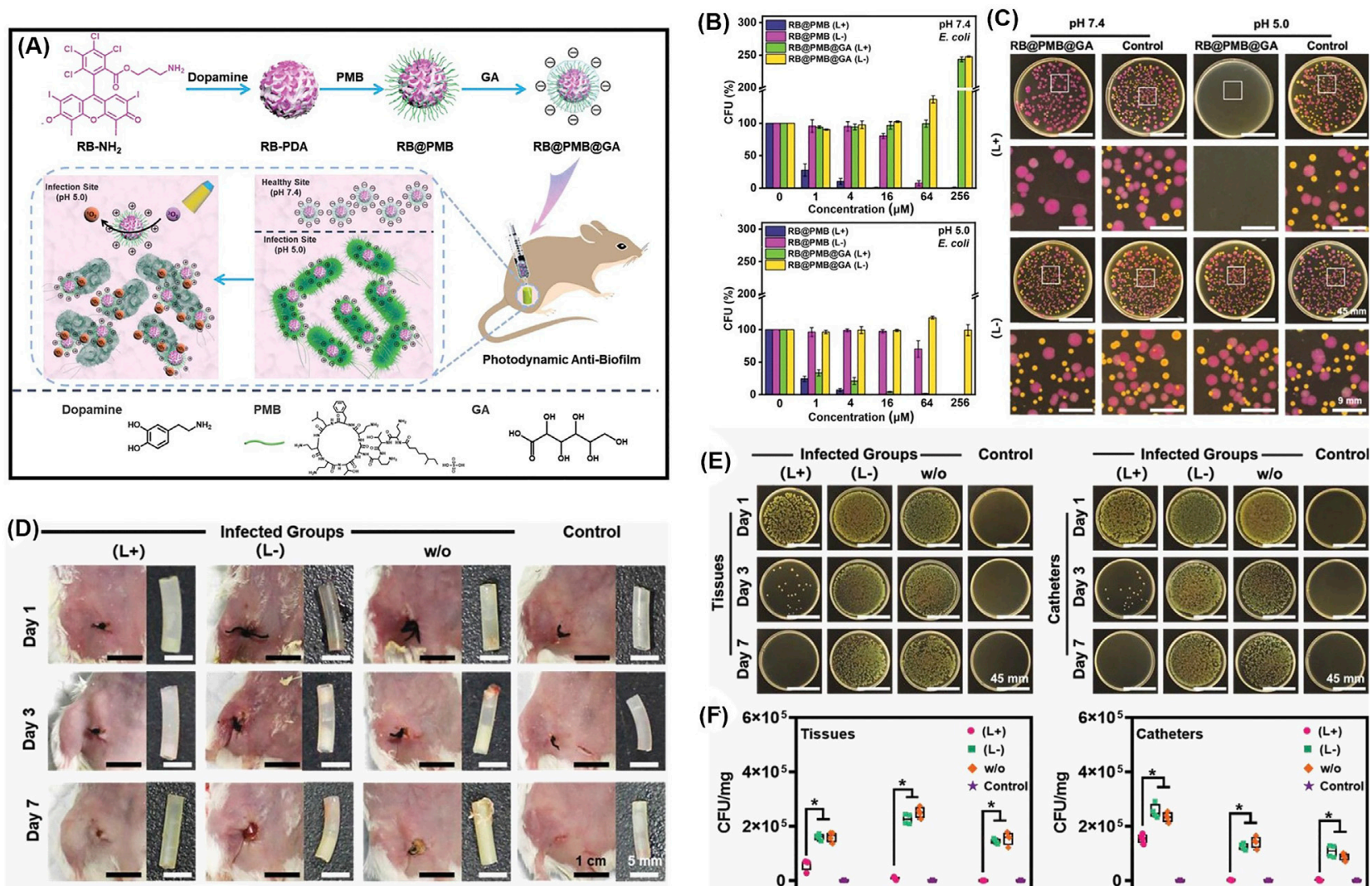
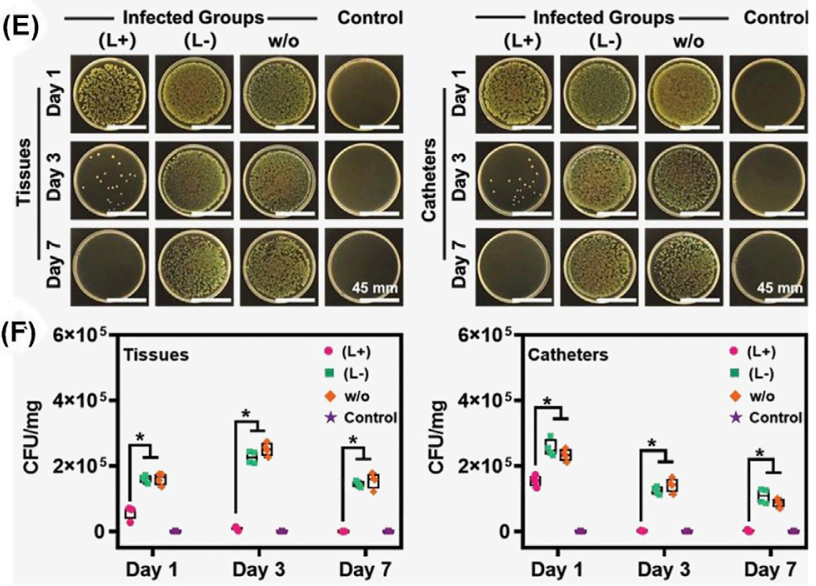

FIGURE 14 | (A) Schematic illustration of the preparation process of photodynamic NPs for enhanced penetration and antibacterial efficiency in biofilms; (B) antibacterial activities against $E$. coli at $\mathrm{pH} 7.4$ and $\mathrm{pH} 5.0$, with or without irradiation; (C) photographs of $E$. coli labeled with the red protein and $S$. aureus colonies incubated together with the different treatments; (D) typical photographs of the incision areas and implanted catheters from mice under different treatments on Days 1 , 3 , and 7 ; (E) photographs of $P$. aeruginosa bacterial colonies, and (F) quantitative analysis of bacterial colony-forming units obtained from the tissues and catheters in each group. Reprinted with permission from Wu et al. (2021).

quenching effect. As a result, enhanced fluorescence emission and singlet oxygen production ability of TAPP were observed in the presence of $\mathrm{H}_{2} \mathrm{O}_{2}$ at $\mathrm{pH}=5.5$. The in vitro antibacterial effect was examined (Figure 15B and Figure 15C). The zeta potential of the bacteria is increased from -10 to $+2 \mathrm{mV}$ when incubated with the nanoparticles, indicating the adherence ability of nanoparticles to bacteria. There was $80 \%$ of $S$. aureus killed by nanoparticles in the presence of $\mathrm{H}_{2} \mathrm{O}_{2}$ upon light irradiation (Figure 15B and Figure 15C). In vivo anti-biofilm activity of nanoparticles was quantified, and an obvious depression of biofilm was observed for the PDT treatment group.

\section{GSH}

Disulfide bond is frequently used as the GSH responsive linker to construct smart polymeric delivery systems for antibacterial PDT (Lu et al., 2018; Ye et al., 2020). For instance, the hyperbranched PEG was loaded with (Zinc)Porphyrins via disulfide and benzacetal linkers, which are sensitive to reductive (GSH) and acidic microenvironment of bacteria, respectively (Staegemann et al., 2018). The release of photosensitizer (Zinc)Porphyrins is confirmed via thin layer chromatography (TLC), size exclusion chromatography (SEC), dialysis and extraction, etc. The polymeric material shows significant phototoxicity against $S$. aureus. The benzacetal linker containing polymeric material was also applied for the PDT of tumor, which shows a less efficient depression of the tumor. This might be due to the more reductive and acidic microenvironment of bacteria than tumor, leading to more complete release of photosensitizers in bacteria and hence a better PDT efficacy. This result indicates that the treatments of cancer and bacteria are different.

\section{SUMMARY AND OUTLOOK}

Photodynamic therapy (PDT) has attracted great attention in the antitumor and antimicrobial area. This review introduces the smart polymeric delivery system for the PDT of tumor and bacterial infections. In particular, targeted and activatable polymeric delivery systems are highlighted. Due to the different structure of tumor and bacterial cells, the target point is different to some extent. For instance, the anti-tumor therapy targets the tumor vasculature, tumor cells, and tumor subcellular 


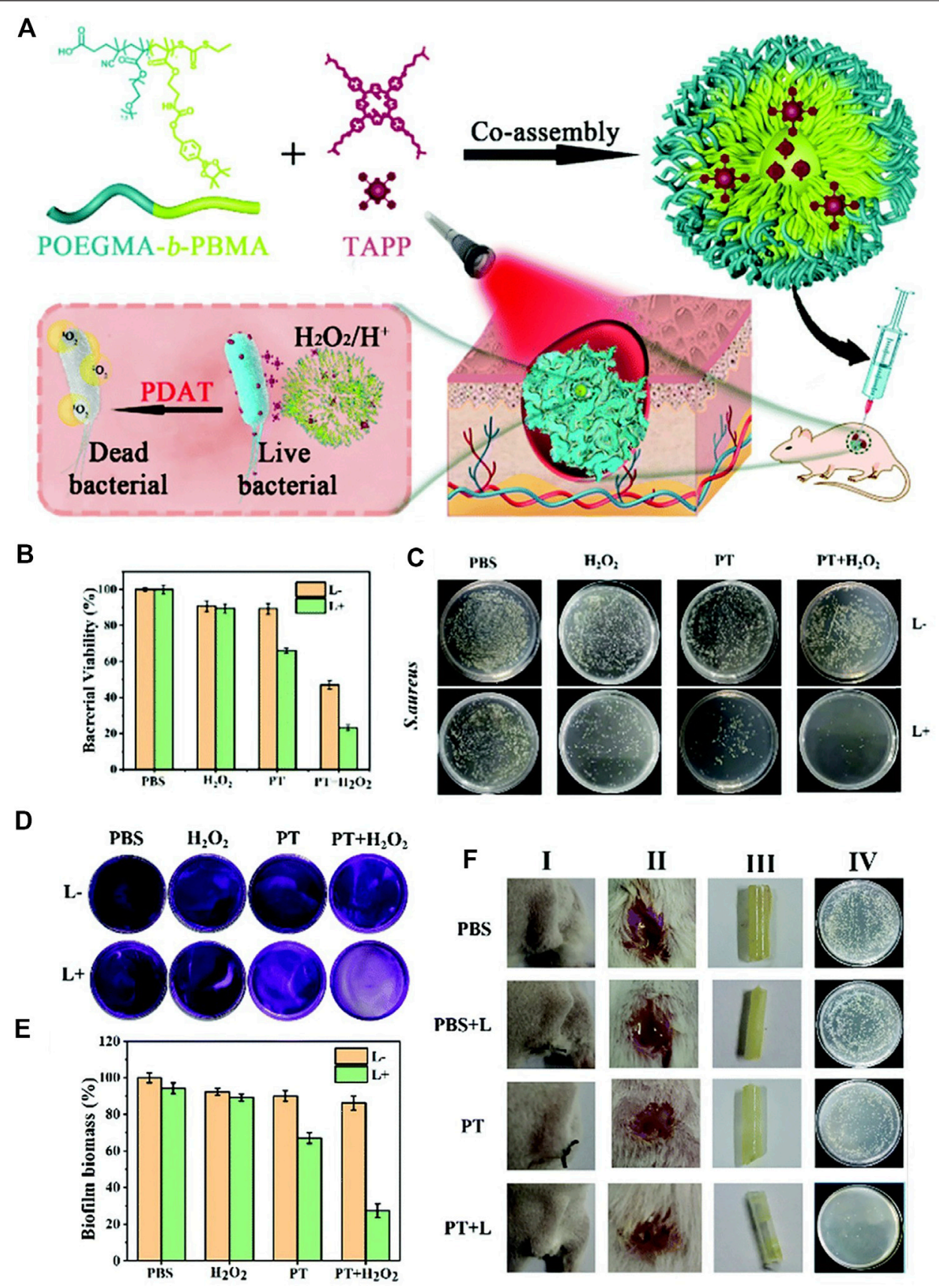

FIGURE 15 | (A) Schematic diagram of a pH/ $\mathrm{H}_{2} \mathrm{O}_{2}$ dual-responsive nanoplatform and its photodynamic antimicrobial therapy process; (B) bacterial viability of S. aureus treated with different conditions at $\mathrm{pH}=5.5$; (C) the corresponding S. aureus on agar plates after different treatments. Reprinted with permission from Zhao et al. (2021).

organelles such as mitochondria, lysosome, nucleus, and endoplasmic reticulum. While the anti-bacterial therapy targets the bacteria membranes, bacterial exopolysaccharides (EPS), and fimbriae, etc. On the other hand, the microenvironment of tumor and bacteria shares plenty of similarities such as hypoxic, acidic, highly reductive and have abundant ROS, etc. Hence, activatable polymeric delivery systems for both tumor and bacteria treatment have been developed. The activation is mainly via the following three strategies: (1) self-quenching and dequenching of photosensitizers due to aggregation and disintegration; (2) utilize another quencher to quench the triplet state of photosensitizer and dequenching upon cleavage of sensitive bonds; and (3) the change of size and surface charge to induce an enhanced internalization and penetration of tumor cells or bacterial cells.

Despite of the vigorous development of smart polymeric delivery system for PDT of tumor and bacteria, there are still some problems. First, there is great concern to the uncertainty of 
the molecular structure of polymers, which leads to the problem of reproducibility. Without a stable production and stable property, it is difficult for polymers to be applied in the clinic.

Second, concerning the passive targeting, recently the EPR effect is becoming a controversial topic and the general applicability of this EPR effect is challenged and still an open question.

Third, concerning the activatable polymeric delivery system, although the microenvironment of tumor/bacteria is different from that of normal tissues, there are still risks of inefficient specificity. For instance, ROS is not only abundant in tumor/ bacteria cells, but also active in inflamed tissues. In addition, normal cells also have acidic subcellular compartments such as endosome and lysosome that are similar to those of cancer cells, which limits the $\mathrm{pH}$-activatable theranostics to some extent.

Last but not the least, the reported polymeric delivery systems are usually complicated, and there is great concern to their toxicities. Developing non-toxic and simple polymeric delivery

\section{REFERENCES}

Agostinis, P., Berg, K., Cengel, K. A., Foster, T. H., Girotti, A. W., Gollnick, S. O., et al. (2011). Photodynamic Therapy of Cancer: an Update. CA: A Cancer J. Clinicians 61 (4), 250-281. doi:10.3322/caac.20114

Bae, B.-C., Li, F., Ling, D., and Na, K. (2010). Self-organized Nanogel from Pullulan/pheophorbide-A Conjugate as a Macromolecular Photodynamic Agent. J. Porphyrins Phthalocyanines 14 (10), 851-858. doi:10.1142/ s1088424610002707

Bae, B.-C., and Na, K. (2010). Self-quenching Polysaccharide-Based Nanogels of Pullulan/folate-Photosensitizer Conjugates for Photodynamic Therapy. Biomaterials 31 (24), 6325-6335. doi:10.1016/j.biomaterials.2010.04.030

Blacha-Grzechnik, A. (2021). New Approach in the Application of Conjugated Polymers: The Light-Activated Source of Versatile Singlet Oxygen Molecule. Materials 14 (5), 1098. doi:10.3390/ma14051098

Braslawsky, G. R., Edson, M. A., Pearce, W., Kaneko, T., and Greenfield, R. S. (1990). Antitumor Activity of Adriamycin (Hydrazone-linked) Immunoconjugates Compared with Free Adriamycin and Specificity of Tumor Cell Killing. Cancer Res. 50 (20), 6608-6614.

Broenstrup, M., Tegge, W., Guerra, G., Höltke, A., Schiller, L., Beutling, U., et al. (2021). Selective Bacterial Targeting and Infection-triggered Release of Antibiotic Colistin Conjugates. Angew. Chem. 60, 17989-17997. doi:10.1002/ anie. 202104921

Brown, S. B., Brown, E. A., and Walker, I. (2004). The Present and Future Role of Photodynamic Therapy in Cancer Treatment. Lancet Oncol. 5 (8), 497-508. doi:10.1016/s1470-2045(04)01529-3

Bugaj, A. M. (2011). Targeted Photodynamic Therapy - a Promising Strategy of Tumor Treatment. Photochem. Photobiol. Sci. 10 (7), 1097-1109. doi:10.1039/ c0pp00147c

Bush, K., Courvalin, P., Dantas, G., Davies, J., Eisenstein, B., Huovinen, P., et al. (2011). Tackling Antibiotic Resistance. Nat. Rev. Microbiol. 9 (12), 894-896. doi:10.1038/nrmicro2693

Caffarel-Salvador, E., Kearney, M.-C., Mairs, R., Gallo, L., Stewart, S., Brady, A., et al. (2015). Methylene Blue-Loaded Dissolving Microneedles: Potential Use in Photodynamic Antimicrobial Chemotherapy of Infected Wounds. Pharmaceutics 7 (4), 397-412. doi:10.3390/pharmaceutics7040397

Celli, J. P., Spring, B. Q., Rizvi, I., Evans, C. L., Samkoe, K. S., Verma, S., et al. (2010). Imaging and Photodynamic Therapy: Mechanisms, Monitoring, and Optimization. Chem. Rev. 110 (5), 2795-2838. doi:10.1021/cr900300p

Chakrabortty, S., Agrawalla, B. K., Stumper, A., Vegi, N. M., Fischer, S., Reichardt, C., et al. (2017). Mitochondria Targeted Protein-Ruthenium Photosensitizer for Efficient Photodynamic Applications. J. Am. Chem. Soc. 139 (6), 2512-2519. doi:10.1021/jacs.6b13399

Chen, H., Li, S., Wu, M., Kenry, Z., Huang, Z., Lee, C. S., et al. (2020). MembraneAnchoring Photosensitizer with Aggregation-Induced Emission Characteristics systems and forward their application from bench to bedside is highly desired.

\section{AUTHOR CONTRIBUTIONS}

ZW conceptualized and wrote the manuscript and BY and FX revised the manuscript. All authors approved the final version of the manuscript.

\section{FUNDING}

ZW thanks the China Postdoctoral Science Foundation (2020M670108) for financial support. FX thanks the Beijing Outstanding Young Scientist Program (Project No. BJJWZYJH01201910010024).

for Combating Multidrug-Resistant Bacteria. Angew. Chem. 132 (2), 642-646. doi:10.1002/ange.201907343

Chen, J., Ding, J., Wang, Y., Cheng, J., Ji, S., Zhuang, X., et al. (2017). Sequentially Responsive Shell-Stacked Nanoparticles for Deep Penetration into Solid Tumors. Adv. Mater. 29 (32), 1701170. doi:10.1002/adma.201701170

Chen, N. H., Couñago, R. M., Djoko, K. Y., Jennings, M. P., Apicella, M. A., Kobe, B., et al. (2013). A Glutathione-dependent Detoxification System Is Required for Formaldehyde Resistance and Optimal Survival ofNeisseria Meningitidisin Biofilms. Antioxid. Redox Signaling 18 (7), 743-755. doi:10.1089/ars.2012.4749

Choi, Y., Weissleder, R., and Tung, C.-H. (2006). Selective Antitumor Effect of Novel Protease-Mediated Photodynamic Agent. Cancer Res. 66 (14), 7225-7229. doi:10.1158/0008-5472.can-06-0448

Cui, D., Huang, J., Zhen, X., Li, J., Jiang, Y., and Pu, K. (2019). A Semiconducting Polymer Nano-prodrug for Hypoxia-Activated Photodynamic Cancer Therapy. Angew. Chem. 131 (18), 5981-5985. doi:10.1002/ange.201814730

Dai, Y., Ma, P. a., Cheng, Z., Kang, X., Zhang, X., Hou, Z., et al. (2012). UpConversion Cell Imaging and $\mathrm{pH}$-Induced Thermally Controlled Drug Release from $\mathrm{NaYF}_{4}: \mathrm{Yb}^{3+} / \mathrm{Er}^{3+} @ H y d r o g e l$ Core-Shell Hybrid Microspheres. ACS Nano 6 (4), 3327-3338. doi:10.1021/nn300303q

Danhier, F., Feron, O., and Préat, V. (2010). To Exploit the Tumor Microenvironment: Passive and Active Tumor Targeting of Nanocarriers for Anti-cancer Drug Delivery. J. Controlled Release 148 (2), 135-146. doi:10.1016/ j.jconrel.2010.08.027

del Carmen, M. G., Rizvi, I., Chang, Y., Moor, A. C. E., Oliva, E., Sherwood, M., et al. (2005). Synergism of Epidermal Growth Factor Receptor-Targeted Immunotherapy with Photodynamic Treatment of Ovarian Cancer In Vivo. J. Natl. Cancer 97 (20), 1516-1524. doi:10.1093/jnci/dji314

Demidova, T. N., and Hamblin, M. R. (2004). Photodynamic Therapy Targeted to Pathogens. Int. J. Immunopathol Pharmacol. 17 (3), 245-254. doi:10.1177/ 039463200401700304

Detty, M. R., Gibson, S. L., and Wagner, S. J. (2004). Current Clinical and Preclinical Photosensitizers for Use in Photodynamic Therapy. J. Med. Chem. 47 (16), 3897-3915. doi:10.1021/jm040074b

Dong, Z., Feng, L., Hao, Y., Chen, M., Gao, M., Chao, Y., et al. (2018). Synthesis of Hollow Biomineralized CaCO3-Polydopamine Nanoparticles for Multimodal Imaging-Guided Cancer Photodynamic Therapy with Reduced Skin Photosensitivity. J. Am. Chem. Soc. 140 (6), 2165-2178. doi:10.1021/ jacs.7b11036

Donnelly, R. F., Morrow, D. I. J., McCrudden, M. T. C., Alkilani, A. Z., VicentePérez, E. M., O’Mahony, C., et al. (2014). Hydrogel-Forming and Dissolving Microneedles for Enhanced Delivery of Photosensitizers and Precursors. Photochem. Photobiol. 90 (3), 641-647. doi:10.1111/php.12209

Du, J.-Z., Du, X.-J., Mao, C.-Q., and Wang, J. (2011). Tailor-Made Dual pHSensitive Polymer-Doxorubicin Nanoparticles for Efficient Anticancer Drug Delivery. J. Am. Chem. Soc. 133 (44), 17560-17563. doi:10.1021/ja207150n 
Du, J.-Z., Sun, T.-M., Song, W.-J., Wu, J., and Wang, J. (2010). A Tumor-AcidityActivated Charge-Conversional Nanogel as an Intelligent Vehicle for Promoted Tumoral-Cell Uptake and Drug Delivery. Angew. Chem. 122 (21), 3703-3708. doi:10.1002/ange.200907210

Ferlay, J., Shin, H.-R., Bray, F., Forman, D., Mathers, C., and Parkin, D. M. (2010). Estimates of Worldwide burden of Cancer in 2008: GLOBOCAN 2008. Int. J. Cancer 127 (12), 2893-2917. doi:10.1002/ijc.25516

Figge, F. H. J., Weiland, G. S., and Manganiello, L. O. J. (1948). Cancer Detection and Therapy. Affinity of Neoplastic, Embryonic, and Traumatized Tissues for Porphyrins and Metalloporphyrins. Exp. Biol. Med. 68 (3), 640-641. doi:10.3181/00379727-68-16580

Flemming, H.-C., Wingender, J., Szewzyk, U., Steinberg, P., Rice, S. A., and Kjelleberg, S. (2016). Biofilms: an Emergent Form of Bacterial Life. Nat. Rev. Microbiol. 14 (9), 563-575. doi:10.1038/nrmicro.2016.94

Fux, C. A., Costerton, J. W., Stewart, P. S., and Stoodley, P. (2005). Survival Strategies of Infectious Biofilms. Trends Microbiol. 13 (1), 34-40. doi:10.1016/ j.tim.2004.11.010

Gales, G. g., Penninckx, M., Block, J.-C., and Leroy, P. (2008). Role of Glutathione Metabolism Status in the Definition of Some Cellular Parameters and Oxidative Stress Tolerance ofSaccharomyces Cerevisiaecells Growing as Biofilms. FEMS Yeast Res. 8 (5), 667-675. doi:10.1111/j.1567-1364.2008.00401.x

Gu, Y., Zhong, Y., Meng, F., Cheng, R., Deng, C., and Zhong, Z. (2013). Acetallinked Paclitaxel Prodrug Micellar Nanoparticles as a Versatile and Potent Platform for Cancer Therapy. Biomacromolecules 14 (8), 2772-2780. doi:10.1021/bm400615n

Guo, L.-Y., Yan, S.-Z., Tao, X., Yang, Q., Li, Q., Wang, T.-S., et al. (2020). Evaluation of Hypocrellin A-Loaded Lipase Sensitive Polymer Micelles for Intervening Methicillin-Resistant Staphylococcus Aureus Antibiotic-Resistant Bacterial Infection. Mater. Sci. Eng. C 106, 110230. doi:10.1016/ j.msec.2019.110230

Hamblin, M. R., and Hasan, T. (2004). Photodynamic Therapy: a New Antimicrobial Approach to Infectious Disease? Photochem. Photobiol. Sci. 3 (5), 436-450. doi:10.1039/b311900a

Han, T., Wang, X., Wang, D., and Tang, B. Z. (2021). Functional Polymer Systems with Aggregation-Induced Emission and Stimuli Responses. Top. Curr. Chem. (Cham) 379 (1), 7-47. doi:10.1007/s41061-020-00321-7

Hanahan, D., and Weinberg, R. A. (2011). Hallmarks of Cancer: the Next Generation. Cell 144 (5), 646-674. doi:10.1016/j.cell.2011.02.013

Henry, S., McAllister, D. V., Allen, M. G., and Prausnitz, M. R. (1998). Microfabricated Microneedles: a Novel Approach to Transdermal Drug Delivery. J. Pharm. Sci. 87 (8), 922-925. doi:10.1021/js980042+

Hoare, T., and Pelton, R. (2004). Highly pH and Temperature Responsive Microgels Functionalized with Vinylacetic Acid. Macromolecules 37 (7), 2544-2550. doi:10.1021/ma035658m

Hoffman, A. S. (2008). The Origins and Evolution of "Controlled" Drug Delivery Systems. J. Controlled Release 132 (3), 153-163. doi:10.1016/ j.jconrel.2008.08.012

Hu, D., Deng, Y., Jia, F., Jin, Q., and Ji, J. (2019). Surface Charge Switchable Supramolecular Nanocarriers for Nitric Oxide Synergistic Photodynamic Eradication of Biofilms. ACS Nano 14 (1), 347-359. doi:10.1021/ acsnano.9b05493

Huang, L., Li, Z., Zhao, Y., Zhang, Y., Wu, S., Zhao, J., et al. (2016). Ultralow-power Near Infrared Lamp Light Operable Targeted Organic Nanoparticle Photodynamic Therapy. J. Am. Chem. Soc. 138 (44), 14586-14591. doi:10.1021/jacs.6b05390

Huang, Y.-Y., Sharma, S. K., Dai, T., Chung, H., Yaroslavsky, A., Garcia-Diaz, M., et al. (2012). Can Nanotechnology Potentiate Photodynamic Therapy? Nanotechnol. Rev. 1 (2), 111-146. doi:10.1515/ntrev-2011-0005

Huo, D., Zhu, J., Chen, G., Chen, Q., Zhang, C., Luo, X., et al. (2019). Eradication of Unresectable Liver Metastasis through Induction of Tumour Specific Energy Depletion. Nat. Commun. 10 (1). doi:10.1038/s41467-019-11082-3

Jiang, B.-P., Zhang, L., Guo, X.-L., Shen, X.-C., Wang, Y., Zhu, Y., et al. (2017). Poly(N-phenylglycine)-Based Nanoparticles as Highly Effective and Targeted Near-Infrared Photothermal Therapy/Photodynamic Therapeutic Agents for Malignant Melanoma. Small 13 (8), 1602496. doi:10.1002/smll.201602496

Jiang, Y., Yang, X., Ma, C., Wang, C., Chen, Y., Dong, F., et al. (2014). Interfacing a Tetraphenylethene Derivative and a Smart Hydrogel for Temperaturedependent Photoluminescence with Sensitive Thermoresponse. ACS Appl. Mater. Inter. 6 (7), 4650-4657. doi:10.1021/am501106x
Kamaly, N., Yameen, B., Wu, J., and Farokhzad, O. C. (2016). Degradable Controlled-Release Polymers and Polymeric Nanoparticles: Mechanisms of Controlling Drug Release. Chem. Rev. 116 (4), 2602-2663. doi:10.1021/ acs.chemrev.5b00346

Kamkaew, A., Lim, S. H., Lee, H. B., Kiew, L. V., Chung, L. Y., and Burgess, K. (2013). BODIPY Dyes in Photodynamic Therapy. Chem. Soc. Rev. 42 (1), 77-88. doi:10.1039/c2cs35216h

Kaspler, P., Lazic, S., Forward, S., Arenas, Y., Mandel, A., and Lilge, L. (2016). A Ruthenium(ii) Based Photosensitizer and Transferrin Complexes Enhance Photo-Physical Properties, Cell Uptake, and Photodynamic Therapy Safety and Efficacy. Photochem. Photobiol. Sci. 15 (4), 481-495. doi:10.1039/ c5pp00450k

Ke, X., Coady, D. J., Yang, C., Engler, A. C., Hedrick, J. L., and Yang, Y. Y. (2014). pH-sensitive Polycarbonate Micelles for Enhanced Intracellular Release of Anticancer Drugs: a Strategy to Circumvent Multidrug Resistance. Polym. Chem. 5 (7), 2621-2628. doi:10.1039/c3py01784b

Kearney, M.-C., Brown, S., McCrudden, M. T. C., Brady, A. J., and Donnelly, R. F. (2014). Potential of Microneedles in Enhancing Delivery of Photosensitising Agents for Photodynamic Therapy. Photodiagnosis Photodynamic Ther. 11 (4), 459-466. doi:10.1016/j.pdpdt.2014.09.003

Keerthiga, R., Zhao, Z., Pei, D., and Fu, A. (2020). Photodynamic Nanophotosensitizers: Promising Materials for Tumor Theranostics. ACS Biomater. Sci. Eng. 6 (10), 5474-5485. doi:10.1021/acsbiomaterials.0c01058

Kim, J., Yoon, H.-J., Kim, S., Wang, K., Ishii, T., Kim, Y.-R., et al. (2009). Polymermetal Complex Micelles for the Combination of Sustained Drug Releasing and Photodynamic Therapy. J. Mater. Chem. 19 (26), 4627-4631. doi:10.1039/ b904224e

Kim, W. L., Cho, H., Li, L., Kang, H. C., and Huh, K. M. (2014). Biarmed Poly(ethylene Glycol)-(pheophorbide A)2 Conjugate as a Bioactivatable Delivery Carrier for Photodynamic Therapy. Biomacromolecules 15 (6), 2224-2234. doi:10.1021/bm5003619

Klare, W., Das, T., Ibugo, A., Buckle, E., Manefield, M., and Manos, J. (2016). Glutathione-disrupted Biofilms of Clinical Pseudomonas aeruginosa Strains Exhibit an Enhanced Antibiotic Effect and a Novel Biofilm Transcriptome. Antimicrob. Agents Chemother. 60 (8), 4539-4551. doi:10.1128/aac.02919-15

Klausen, M., Ucuncu, M., and Bradley, M. (2020). Design of Photosensitizing Agents for Targeted Antimicrobial Photodynamic Therapy. Molecules 25 (22), 5239. doi:10.3390/molecules 25225239

Koo, H., Allan, R. N., Howlin, R. P., Stoodley, P., and Hall-Stoodley, L. (2017). Targeting Microbial Biofilms: Current and Prospective Therapeutic Strategies. Nat. Rev. Microbiol. 15 (12), 740-755. doi:10.1038/nrmicro.2017.99

Krogfelt, K. A., Bergmans, H., and Klemm, P. (1990). Direct Evidence that the FimH Protein Is the Mannose-specific Adhesin of Escherichia coli Type 1 Fimbriae. Infect. Immun. 58 (6), 1995-1998. doi:10.1128/iai.58.6.19951998.1990

Lambert, P. A. (2002). Cellular Impermeability and Uptake of Biocides and Antibiotics in Gram-Positive Bacteria and Mycobacteria. J. Appl. Microbiol. 92, 46S-54S. doi:10.1046/j.1365-2672.92.5s1.7.x

Lee, J. W., Park, J.-H., and Prausnitz, M. R. (2008). Dissolving Microneedles for Transdermal Drug Delivery. Biomaterials 29 (13), 2113-2124. doi:10.1016/ j.biomaterials.2007.12.048

Lee, S. J., Park, K., Oh, Y.-K., Kwon, S.-H., Her, S., Kim, I.-S., et al. (2009). Tumor Specificity and Therapeutic Efficacy of Photosensitizer-Encapsulated Glycol Chitosan-Based Nanoparticles in Tumor-Bearing Mice. Biomaterials 30 (15), 2929-2939. doi:10.1016/j.biomaterials.2009.01.058

Li, C., Lin, F., Sun, W., Wu, F.-G., Yang, H., Lv, R., et al. (2018a). Self-assembled Rose Bengal-Exopolysaccharide Nanoparticles for Improved Photodynamic Inactivation of Bacteria by Enhancing Singlet Oxygen Generation Directly in the Solution. ACS Appl. Mater. Inter. 10 (19), 16715-16722. doi:10.1021/ acsami.8b01545

Li, F., Bae, B.-c., and Na, K. (2010). Acetylated Hyaluronic Acid/Photosensitizer Conjugate for the Preparation of Nanogels with Controllable Phototoxicity: Synthesis, Characterization, Autophotoquenching Properties, Andin vitroPhototoxicity against HeLa Cells. Bioconjug. Chem. 21 (7), 1312-1320. doi:10.1021/bc100116v

Li, F., Chen, C., Yang, X., He, X., Zhao, Z., Li, J., et al. (2018b). Acetal-linked hyperbranched polyphosphoester nanocarriers loaded with chlorin e6 for $\mathrm{pH}$ - 
activatable photodynamic therapy. ACS Appl. Mater. Inter. 10 (25), 21198-21205. doi:10.1021/acsami.8b06758

Li, H., Wang, P., Deng, Y., Zeng, M., Tang, Y., Zhu, W.-H., et al. (2017a). Combination of Active Targeting, Enzyme-Triggered Release and Fluorescent Dye into Gold Nanoclusters for Endomicroscopy-Guided Photothermal/photodynamic Therapy to Pancreatic Ductal Adenocarcinoma. Biomaterials 139, 30-38. doi:10.1016/ j.biomaterials.2017.05.030

Li, J., Cui, D., Huang, J., He, S., Yang, Z., Zhang, Y., et al. (2019a). Organic Semiconducting Pro-nanostimulants for Near-Infrared Photoactivatable Cancer Immunotherapy. Angew. Chem. 131 (36), 12810-12817. doi:10.1002/ ange.201906288

Li, J., Cui, D., Jiang, Y., Huang, J., Cheng, P., and Pu, K. (2019b). Near-Infrared Photoactivatable Semiconducting Polymer Nanoblockaders for MetastasisInhibited Combination Cancer Therapy. Adv. Mater. 31 (46), 1905091. doi:10.1002/adma.201905091

Li, J., Huang, J., Lyu, Y., Huang, J., Jiang, Y., Xie, C., et al. (2019c). Photoactivatable Organic Semiconducting Pro-nanoenzymes. J. Am. Chem. Soc. 141 (9), 4073-4079. doi:10.1021/jacs.8b13507

Li, J., Meng, X., Deng, J., Lu, D., Zhang, X., Chen, Y., et al. (2018c). Multifunctional Micelles Dually Responsive to Hypoxia and Singlet Oxygen: Enhanced Photodynamic Therapy via Interactively Triggered Photosensitizer Delivery. ACS Appl. Mater. Inter. 10 (20), 17117-17128. doi:10.1021/acsami.8b06299

$\mathrm{Li}, \mathrm{J}$., and Pu, K. (2020). Semiconducting Polymer Nanomaterials as Near-Infrared Photoactivatable Protherapeutics for Cancer. Acc. Chem. Res. 53 (4), 752-762. doi:10.1021/acs.accounts.9b00569

Li, J., Yao, S., Wang, K., Lu, Z., Su, X., Li, L., et al. (2018d). Hypocrellin B-Loaded, Folate-Conjugated Polymeric Micelle for Intraperitoneal Targeting of Ovarian Cancer In Vitro and In Vivo. Cancer Sci. 109 (6), 1958-1969. doi:10.1111/ cas. 13605

Li, L., and Huh, K. M. (2014). Polymeric Nanocarrier Systems for Photodynamic Therapy. Biomater. Res. 18 (1), 19-14. doi:10.1186/2055-7124-18-19

Li, L., Bae, B.-c., Tran, T. H., Yoon, K. H., Na, K., and Huh, K. M. (2011). Selfquenchable Biofunctional Nanoparticles of Heparin-Folate-Photosensitizer Conjugates for Photodynamic Therapy. Carbohydr. Polym. 86 (2), 708-715. doi:10.1016/j.carbpol.2011.05.011

Li, L., Nurunnabi, M., Nafiujjaman, M., Jeong, Y. Y., Lee, Y.-k., and Huh, K. M. (2014). A Photosensitizer-Conjugated Magnetic Iron Oxide/gold Hybrid Nanoparticle as an Activatable Platform for Photodynamic Cancer Therapy. J. Mater. Chem. B 2 (19), 2929-2937. doi:10.1039/c4tb00181h

Li, T., and Yan, L. (2018). Functional Polymer Nanocarriers for Photodynamic Therapy. Pharmaceuticals 11 (4), 133. doi:10.3390/ph11040133

Li, X., Kim, J., Yoon, J., and Chen, X. (2017b). Cancer-Associated, Stimuli-Driven, Turn on Theranostics for Multimodality Imaging and Therapy. Adv. Mater. 29 (23), 1606857. doi:10.1002/adma.201606857

Li, Y., Wu, Q., Kang, M., Song, N., Wang, D., and Tang, B. Z. (2020). Boosting the Photodynamic Therapy Efficiency by Using Stimuli-Responsive and AIEFeatured Nanoparticles. Biomaterials 232, 119749. doi:10.1016/ j.biomaterials.2019.119749

Lin, A.-L., Li, S.-Z., Xu, C.-H., Li, X.-S., Zheng, B.-Y., Gu, J.-J., et al. (2019). A pHResponsive Stellate Mesoporous Silica Based Nanophotosensitizer for In Vivo Cancer Diagnosis and Targeted Photodynamic Therapy. Biomater. Sci. 7 (1), 211-219. doi:10.1039/c8bm00386f

Lincoln, R., Kohler, L., Monro, S., Yin, H., Stephenson, M., Zong, R., et al. (2013). Exploitation of Long-Lived 3IL Excited States for Metal-Organic Photodynamic Therapy: Verification in a Metastatic Melanoma Model. J. Am. Chem. Soc. 135 (45), 17161-17175. doi:10.1021/ja408426z

Liu, C., Guo, J., Yang, W., Hu, J., Wang, C., and Fu, S. (2009). Magnetic Mesoporous Silica Microspheres with Thermo-Sensitive Polymer Shell for Controlled Drug Release. J. Mater. Chem. 19 (27), 4764-4770. doi:10.1039/ b902985k

Liu, L.-H., Qiu, W.-X., Zhang, Y.-H., Li, B., Zhang, C., Gao, F., et al. (2017). A Charge Reversible Self-Delivery Chimeric Peptide with Cell MembraneTargeting Properties for Enhanced Photodynamic Therapy. Adv. Funct. Mater. 27 (25), 1700220. doi:10.1002/adfm.201700220

Liu, L., Ruan, Z., Li, T., Yuan, P., and Yan, L. (2016a). Near Infrared ImagingGuided Photodynamic Therapy under an Extremely Low Energy of Light by
Galactose Targeted Amphiphilic Polypeptide Micelle Encapsulating BODIPYBr2. Biomater. Sci. 4 (11), 1638-1645. doi:10.1039/c6bm00581k

Liu, S., Feng, G., Tang, B. Z., and Liu, B. (2021). Recent Advances of AIE Light-Up Probes for Photodynamic Therapy. Chem. Sci. 12 (19), 6488-6506. doi:10.1039/d1sc00045d

Liu, T. W., Akens, M. K., Chen, J., Wilson, B. C., and Zheng, G. (2016b). Matrix Metalloproteinase-Based Photodynamic Molecular Beacons for Targeted Destruction of Bone Metastases In Vivo. Photochem. Photobiol. Sci. 15 (3), 375-381. doi:10.1039/c5pp00414d

Liu, X., Wu, M., Hu, Q., Bai, H., Zhang, S., Shen, Y., et al. (2016c). Redox-activated Light-Up Nanomicelle for Precise Imaging-Guided Cancer Therapy and RealTime Pharmacokinetic Monitoring. ACS Nano 10 (12), 11385-11396. doi:10.1021/acsnano.6b06688

Lu, L., Zhao, X., Fu, T., Li, K., He, Y., Luo, Z., et al. (2020a). An iRGD-Conjugated Prodrug Micelle with Blood-Brain-Barrier Penetrability for Anti-glioma Therapy. Biomaterials 230, 119666. doi:10.1016/j.biomaterials.2019.119666

Lu, M.-m., Ge, Y., Qiu, J., Shao, D., Zhang, Y., Bai, J., et al. (2018). Redox/pH DualControlled Release of Chlorhexidine and Silver Ions from Biodegradable Mesoporous Silica Nanoparticles against Oral Biofilms. Ijn 13, 7697-7709. doi:10.2147/ijn.s181168

Lu, Y., Song, G., He, B., Zhang, H., Wang, X., Zhou, D., et al. (2020b). Strengthened Tumor Photodynamic Therapy Based on a Visible Nanoscale Covalent Organic Polymer Engineered by Microwave Assisted Synthesis. Adv. Funct. Mater. 30 (45), 2004834. doi:10.1002/adfm.202004834

Lucky, S. S., Soo, K. C., and Zhang, Y. (2015). Nanoparticles in Photodynamic Therapy. Chem. Rev. 115 (4), 1990-2042. doi:10.1021/cr5004198

Lv, R., Yang, P., He, F., Gai, S., Yang, G., Dai, Y., et al. (2015). An Imaging-Guided Platform for Synergistic Photodynamic/photothermal/chemo-Therapy with $\mathrm{pH} /$ temperature-Responsive Drug Release. Biomaterials 63, 115-127. doi:10.1016/j.biomaterials.2015.05.016

Maisch, T., Szeimies, R.-M., Jori, G., and Abels, C. (2004). Antibacterial Photodynamic Therapy in Dermatology. Photochem. Photobiol. Sci. 3 (10), 907-917. doi:10.1039/b407622b

Majumdar, P., Nomula, R., and Zhao, J. (2014). Activatable Triplet Photosensitizers: Magic Bullets for Targeted Photodynamic Therapy. J. Mater. Chem. C 2 (30), 5982-5997. doi:10.1039/c4tc00659c

Malik, Z., Ladan, H., and Nitzan, Y. (1992). Photodynamic Inactivation of GramNegative Bacteria: Problems and Possible Solutions. J. Photochem. Photobiol. B: Biol. 14 (3), 262-266. doi:10.1016/1011-1344(92)85104-3

Minnock, A., Vernon, D. I., Schofield, J., Griffiths, J., Howard Parish, J., and Brown, S. B. (1996). Photoinactivation of Bacteria. Use of a Cationic Water-Soluble Zinc Phthalocyanine to Photoinactivate Both Gram-Negative and GramPositive Bacteria. J. Photochem. Photobiol. B: Biol. 32 (3), 159-164. doi:10.1016/1011-1344(95)07148-2

Minnock, A., Vernon, D. I., Schofield, J., Griffiths, J., Parish, J. H., and Brown, S. B. (2000). Mechanism of Uptake of a Cationic Water-Soluble Pyridinium Zinc Phthalocyanine across the Outer Membrane of Escherichia coli. Antimicrob. Agents Chemother. 44 (3), 522-527. doi:10.1128/aac.44.3.522-527.2000

Mitsunaga, M., Ogawa, M., Kosaka, N., Rosenblum, L. T., Choyke, P. L., and Kobayashi, H. (2011). Cancer Cell-Selective In Vivo Near Infrared Photoimmunotherapy Targeting Specific Membrane Molecules. Nat. Med. 17 (12), 1685-1691. doi:10.1038/nm.2554

Oh, I.-h., Min, H. S., Li, L., Tran, T. H., Lee, Y.-k., Kwon, I. C., et al. (2013). Cancer Cell-specific Photoactivity of Pheophorbide A-Glycol Chitosan Nanoparticles for Photodynamic Therapy in Tumor-Bearing Mice. Biomaterials 34 (27), 6454-6463. doi:10.1016/j.biomaterials.2013.05.017

Pagonis, T. C., Chen, J., Fontana, C. R., Devalapally, H., Ruggiero, K., Song, X., et al. (2010). Nanoparticle-based Endodontic Antimicrobial Photodynamic Therapy. J. Endodontics 36 (2), 322-328. doi:10.1016/j.joen.2009.10.011

Pan, L., Liu, J., He, Q., Wang, L., and Shi, J. (2013). Overcoming Multidrug Resistance of Cancer Cells by Direct Intranuclear Drug Delivery Using TATConjugated Mesoporous Silica Nanoparticles. Biomaterials 34 (11), 2719-2730. doi:10.1016/j.biomaterials.2012.12.040

Park, S. Y., Baik, H. J., Oh, Y. T., Oh, K. T., Youn, Y. S., and Lee, E. S. (2011a). A Smart Polysaccharide/drug Conjugate for Photodynamic Therapy. Angew. Chem. Int. Ed. 50 (7), 1644-1647. doi:10.1002/anie.201006038

Park, S. Y., Lee, H. U., Lee, Y.-C., Kim, G. H., Park, E. C., Han, S. H., et al. (2014). Wound Healing Potential of Antibacterial Microneedles Loaded 
with green tea Extracts. Mater. Sci. Eng. C 42, 757-762. doi:10.1016/ j.msec.2014.06.021

Park, W., Bae, B.-c., and Na, K. (2016). A Highly Tumor-specific Light-Triggerable Drug Carrier Responds to Hypoxic Tumor Conditions for Effective Tumor Treatment. Biomaterials 77, 227-234. doi:10.1016/j.biomaterials.2015.11.014

Park, W., Park, S.-j., and Na, K. (2011b). The Controlled Photoactivity of Nanoparticles Derived from Ionic Interactions between a Water Soluble Polymeric Photosensitizer and Polysaccharide Quencher. Biomaterials 32 (32), 8261-8270. doi:10.1016/j.biomaterials.2011.07.023

Pelton, R. H., and Chibante, P. (1986). Preparation of Aqueous Latices with N-Isopropylacrylamide. Colloids Surf. 20 (3), 247-256. doi:10.1016/01666622(86)80274-8

Pelton, R. (2000). Temperature-sensitive Aqueous Microgels. Adv. Colloid Interf. Sci. 85 (1), 1-33. doi:10.1016/s0001-8686(99)00023-8

Prasad, P., Gordijo, C. R., Abbasi, A. Z., Maeda, A., Ip, A., Rauth, A. M., et al. (2014). Multifunctional Albumin-MnO2 Nanoparticles Modulate Solid Tumor Microenvironment by Attenuating Hypoxia, Acidosis, Vascular Endothelial Growth Factor and Enhance Radiation Response. ACS Nano 8 (4), 3202-3212. doi:10.1021/nn405773r

Radovic-Moreno, A. F., Lu, T. K., Puscasu, V. A., Yoon, C. J., Langer, R., and Farokhzad, O. C. (2012). Surface Charge-Switching Polymeric Nanoparticles for Bacterial Cell wall-targeted Delivery of Antibiotics. ACS Nano 6 (5), 4279-4287. doi:10.1021/nn3008383

Regehly, M., Greish, K., Rancan, F., Maeda, H., Böhm, F., and Röder, B. (2007). Water-soluble Polymer Conjugates of ZnPP for Photodynamic Tumor Therapy. Bioconjug. Chem. 18 (2), 494-499. doi:10.1021/bc060158u

Rijcken, C. J. F., Hofman, J.-W., van Zeeland, F., Hennink, W. E., and van Nostrum, C. F. (2007). Photosensitiser-loaded Biodegradable Polymeric Micelles: Preparation, Characterisation and In Vitro PDT Efficacy. J. Controlled Release 124 (3), 144-153. doi:10.1016/j.jconrel.2007.09.002

Saravanakumar, G., Kim, J., and Kim, W. J. (2017). Reactive-Oxygen-SpeciesResponsive Drug Delivery Systems: Promises and Challenges. Adv. Sci. 4 (1), 1600124. doi:10.1002/advs.201600124

Sarkisyan, K. S., Zlobovskaya, O. A., Gorbachev, D. A., Bozhanova, N. G., Sharonov, G. V., Staroverov, D. B., et al. (2015). KillerOrange, a Genetically Encoded Photosensitizer Activated by Blue and green Light. PLoS One 10 (12), e0145287. doi:10.1371/journal.pone.0145287

Staegemann, M. H., Gräfe, S., Gitter, B., Achazi, K., Quaas, E., Haag, R., et al. (2018). Hyperbranched Polyglycerol Loaded with (Zinc-)Porphyrins: Photosensitizer Release under Reductive and Acidic Conditions for Improved Photodynamic Therapy. Biomacromolecules 19 (1), 222-238. doi:10.1021/acs.biomac.7b01485

Stöber, W., Fink, A., and Bohn, E. (1968). Controlled Growth of Monodisperse Silica Spheres in the Micron Size Range. J. Colloid Interf. Sci. 26 (1), 62-69. doi:10.1016/0021-9797(68)90272-5

Sun, C., Ji, S., Li, F., and Xu, H. (2017). Diselenide-containing Hyperbranched Polymer with Light-Induced Cytotoxicity. ACS Appl. Mater. Inter. 9 (15), 12924-12929. doi:10.1021/acsami.7b02367

Tian, J., Ding, L., Xu, H.-J., Shen, Z., Ju, H., Jia, L., et al. (2013). Cell-specific and pH-Activatable Rubyrin-Loaded Nanoparticles for Highly Selective NearInfrared Photodynamic Therapy against Cancer. J. Am. Chem. Soc. 135 (50), 18850-18858. doi:10.1021/ja408286k

Tirand, L., Frochot, C., Vanderesse, R., Thomas, N., Trinquet, E., Pinel, S., et al. (2006). A Peptide Competing with VEGF165 Binding on Neuropilin-1 Mediates Targeting of a Chlorin-type Photosensitizer and Potentiates its Photodynamic Activity in Human Endothelial Cells. J. Control Release 111 (1-2), 153-164. doi:10.1016/j.jconrel.2005.11.017

Tseng, S. J., Liao, Z. X., Kao, S. H., Zeng, Y. F., Huang, K. Y., Li, H. J., et al. (2015). Highly Specific In Vivo Gene Delivery for P53-Mediated Apoptosis and Genetic Photodynamic Therapies of Tumour. Nat. Commun. 6 (1). doi:10.1038/ncomms7456

Tuan-Mahmood, T.-M., McCrudden, M. T. C., Torrisi, B. M., McAlister, E., Garland, M. J., Singh, T. R. R., et al. (2013). Microneedles for Intradermal and Transdermal Drug Delivery. Eur. J. Pharm. Sci. 50 (5), 623-637. doi:10.1016/j.ejps.2013.05.005

Vert, M., Li, S. M., Spenlehauer, G., and Guérin, P. (1992). Bioresorbability and Biocompatibility of Aliphatic Polyesters. J. Mater. Sci. Mater. Med. 3 (6), 432-446. doi:10.1007/bf00701240
Wang, C., Cheng, L., Liu, Y., Wang, X., Ma, X., Deng, Z., et al. (2013). ImagingGuided pH-Sensitive Photodynamic Therapy Using Charge Reversible Upconversion Nanoparticles under Near-Infrared Light. Adv. Funct. Mater. 23 (24), 3077-3086. doi:10.1002/adfm.201202992

Wang, M., Zhai, Y., Ye, H., Lv, Q., Sun, B., Luo, C., et al. (2019). High Co-loading Capacity and Stimuli-Responsive Release Based on cascade Reaction of SelfDestructive Polymer for Improved Chemo-Photodynamic Therapy. ACS Nano 13 (6), 7010-7023. doi:10.1021/acsnano.9b02096

Wang, X., and Guo, Z. (2013). Targeting and Delivery of Platinum-Based Anticancer Drugs. Chem. Soc. Rev. 42 (1), 202-224. doi:10.1039/c2cs35259a

Wang, Z., Huang, L., Yan, Y., El-Zohry, A. M., Toffoletti, A., Zhao, J., et al. (2020). Elucidation of the Intersystem Crossing Mechanism in a Helical BODIPY for Low-Dose Photodynamic Therapy. Angew. Chem. 132 (37), 16248-16255. doi:10.1002/ange.202005269

Wei, X., Liu, L., Guo, X., Wang, Y., Zhao, J., and Zhou, S. (2018). Light-activated ROS-Responsive Nanoplatform Codelivering Apatinib and Doxorubicin for Enhanced Chemo-Photodynamic Therapy of Multidrug-Resistant Tumors. ACS Appl. Mater. Inter. 10 (21), 17672-17684. doi:10.1021/acsami.8b04163

Wu, L., Sun, Y., Sugimoto, K., Luo, Z., Ishigaki, Y., Pu, K., et al. (2018a). Engineering of Electrochromic Materials as Activatable Probes for Molecular Imaging and Photodynamic Therapy. J. Am. Chem. Soc. 140 (47), 16340-16352. doi:10.1021/jacs.8b10176

Wu, S., Xu, C., Zhu, Y., Zheng, L., Zhang, L., Hu, Y., et al. (2021). Biofilm-Sensitive Photodynamic Nanoparticles for Enhanced Penetration and Antibacterial Efficiency. Adv. Funct. Mater. 31, 2103591. doi:10.1002/adfm.202103591

Wu, W., Mao, D., Cai, X., Duan, Y., Hu, F., Kong, D., et al. (2018b). ONOO- and ClO- Responsive Organic Nanoparticles for Specific In Vivo Image-Guided Photodynamic Bacterial Ablation. Chem. Mater. 30 (11), 3867-3873. doi:10.1021/acs.chemmater.8b01320

Wu, W., Mao, D., Xu, S., Kenry, F., Hu, F., Li, X., et al. (2018c). Polymerizationenhanced Photosensitization. Chem 4 (8), 1937-1951. doi:10.1016/ j.chempr.2018.06.003

Xu, J., Yang, D., Lv, R., Liu, B., Gai, S., He, F., et al. (2016). Design, Fabrication, Luminescence and Biomedical Applications of UCNPs@mSiO2-ZnPc-CDsP(NIPAm-MAA) Nanocomposites. J. Mater. Chem. B 4 (35), 5883-5894. doi:10.1039/c6tb01677d

Yan, J., Wu, Q., Zhao, Z., Wu, J., Ye, H., Liang, Q., et al. (2020). Light-assisted Hierarchical Intratumoral Penetration and Programmed Antitumor Therapy Based on Tumor Microenvironment (TME)-amendatory and Self-Adaptive Polymeric Nanoclusters. Biomaterials 255, 120166. doi:10.1016/ j.biomaterials.2020.120166

Yang, J., Pan, S., Gao, S., Li, T., and Xu, H. (2021). CO/chemosensitization/ antiangiogenesis Synergistic Therapy with H2O2-Responsive DiselenideContaining Polymer. Biomaterials 271, 120721. doi:10.1016/ j.biomaterials.2021.120721

Ye, M., Zhao, Y., Wang, Y., Yodsanit, N., Xie, R., and Gong, S. (2020). pHResponsive Polymer-Drug Conjugate: An Effective Strategy to Combat the Antimicrobial Resistance. Adv. Funct. Mater. 30 (39), 2002655. doi:10.1002/ adfm. 202002655

Ye, M., Zhao, Y., Wang, Y., Zhao, M., Yodsanit, N., Xie, R., et al. (2021). A DualResponsive Antibiotic-Loaded Nanoparticle Specifically Binds Pathogens and Overcomes Antimicrobial-Resistant Infections. Adv. Mater. 33 (9), 2006772. doi:10.1002/adma.202006772

Yin, H., Shi, X., Wang, H., Jin, W., Li, Y., and Fu, Y. (2017). Photodynamic Therapy Targeting VCAM-1-Expressing Human Umbilical Vein Endothelial Cells Using a PpIX-VCAM-1 Binding Peptide-Quantum Dot Conjugate. RSC Adv. 7 (80), 50562-50570. doi:10.1039/c7ra10648c

Yin, R., Agrawal, T., Khan, U., Gupta, G. K., Rai, V., Huang, Y.-Y., et al. (2015). Antimicrobial Photodynamic Inactivation in Nanomedicine: Small Light Strides against Bad Bugs. Nanomedicine 10 (15), 2379-2404. doi:10.2217/ nnm.15.67

Yoon, H. Y., Koo, H., Choi, K. Y., Lee, S. J., Kim, K., Kwon, I. C., et al. (2012). Tumor-targeting Hyaluronic Acid Nanoparticles for Photodynamic Imaging and Therapy. Biomaterials 33 (15), 3980-3989. doi:10.1016/ j.biomaterials.2012.02.016

Yuan, Y.-Y., Mao, C.-Q., Du, X.-J., Du, J.-Z., Wang, F., and Wang, J. (2012). Surface Charge Switchable Nanoparticles Based on Zwitterionic Polymer for Enhanced 
Drug Delivery to Tumor. Adv. Mater. 24 (40), 5476-5480. doi:10.1002/ adma.201202296

Yuan, Y., Liu, J., and Liu, B. (2014). Conjugated-Polyelectrolyte-Based Polyprodrug: Targeted and Image-Guided Photodynamic and Chemotherapy with On-Demand Drug Release upon Irradiation with a Single Light Source. Angew. Chem. Int. Ed. 53 (28), 7163-7168. doi:10.1002/anie.201402189

Zeng, L., Cheng, H., Dai, Y., Su, Z., Wang, C., Lei, L., et al. (2020). In Vivo Regenerable Cerium Oxide Nanozyme-Loaded pH/H2O2-Responsive Nanovesicle for TumorTargeted Photothermal and Photodynamic Therapies. ACS Appl. Mater. Inter. 13 (1), 233-244. doi:10.1021/acsami.0c19074

Zhang, C., Chen, W., Zhang, T., Jiang, X., and Hu, Y. (2020). Hybrid Nanoparticle Composites Applied to Photodynamic Therapy: Strategies and Applications. J. Mater. Chem. B 8 (22), 4726-4737. doi:10.1039/d0tb00093k

Zhang, X., Yang, P., Dai, Y., Ma, P. a., Li, X., Cheng, Z., et al. (2013). Multifunctional Up-Converting Nanocomposites with Smart Polymer Brushes Gated Mesopores for Cell Imaging and Thermo/pH DualResponsive Drug Controlled Release. Adv. Funct. Mater. 23 (33), 4067-4078. doi:10.1002/adfm.201300136

Zhao, Y., Yu, C., Yu, Y., Wei, X., Duan, X., Dai, X., et al. (2019). Bioinspired Heteromultivalent Ligand-Decorated Nanotherapeutic for Enhanced Photothermal and Photodynamic Therapy of Antibiotic-Resistant Bacterial Pneumonia. ACS Appl. Mater. Inter. 11 (43), 39648-39661. doi:10.1021/acsami.9b15118

Zhao, Y., Zhu, Y., Yang, G., Xia, L., Yu, F., Chen, C., et al. (2021). A pH/H2O2 Dual Triggered Nanoplatform for Enhanced Photodynamic Antibacterial Efficiency. J. Mater. Chem. B 9, 5076-5082. doi:10.1039/d1tb00441g

Zheng, G., Chen, J., Stefflova, K., Jarvi, M., Li, H., and Wilson, B. C. (2007). Photodynamic Molecular beacon as an Activatable Photosensitizer Based on Protease-Controlled Singlet Oxygen Quenching and Activation. Proc. Natl. Acad. Sci. 104 (21), 8989-8994. doi:10.1073/pnas.0611142104

Zheng, Y., Li, Z., Chen, H., and Gao, Y. (2020). Nanoparticle-based Drug Delivery Systems for Controllable Photodynamic Cancer Therapy. Eur. J. Pharm. Sci. 144, 105213. doi:10.1016/j.ejps.2020.105213

Zhu, S., Hong, M., Tang, G., Qian, L., Lin, J., Jiang, Y., et al. (2010). Partly PEGylated Polyamidoamine Dendrimer for Tumor-Selective Targeting of Doxorubicin: the Effects of PEGylation Degree and Drug Conjugation Style. Biomaterials 31 (6), 1360-1371. doi:10.1016/ j.biomaterials.2009.10.044

Conflict of Interest: The authors declare that the research was conducted in the absence of any commercial or financial relationships that could be construed as a potential conflict of interest.

Publisher's Note: All claims expressed in this article are solely those of the authors and do not necessarily represent those of their affiliated organizations, or those of the publisher, the editors, and the reviewers. Any product that may be evaluated in this article, or claim that may be made by its manufacturer, is not guaranteed or endorsed by the publisher.

Copyright (C) 2021 Wang, Xu and Yu. This is an open-access article distributed under the terms of the Creative Commons Attribution License (CC BY). The use, distribution or reproduction in other forums is permitted, provided the original author(s) and the copyright owner(s) are credited and that the original publication in this journal is cited, in accordance with accepted academic practice. No use, distribution or reproduction is permitted which does not comply with these terms. 